\title{
ALMA reveals the magnetic field evolution in the high-mass star forming complex G9.62+0.19
}

\author{
D. Dall'Olio ${ }^{1}$, W. H. T. Vlemmings ${ }^{1}$, M. V. Persson ${ }^{1}$, F. O. Alves ${ }^{2}$, H. Beuther ${ }^{3}$, J. M. Girart ${ }^{4,5}$, G. Surcis ${ }^{6}$, \\ J. M. Torrelles ${ }^{4,5}$, and H. J. Van Langevelde ${ }^{7,8}$ \\ ${ }^{1}$ Department of Space, Earth and Environment, Chalmers University of Technology, Onsala Space Observatory, \\ Observatorievägen 90, 43992 Onsala, Sweden \\ e-mail: daria.dallolio@chalmers.se \\ ${ }^{2}$ Max-Planck-Institut für extraterrestrische Physik, Giessenbachstr. 1, 85748 Garching, Germany \\ ${ }^{3}$ Max-Planck-Institute for Astronomy, Königstuhl 17, 69117 Heidelberg, Germany \\ ${ }^{4}$ Institut de Ciències de l'Espai (ICE, CSIC), Can Magrans, s/n, 08193 Cerdanyola del Vallès, Spain \\ ${ }^{5}$ Institut d'Estudis Espacials de Catalunya (IEEC), 08034 Barcelona, Spain \\ ${ }^{6}$ INAF - Osservatorio Astronomico di Cagliari, Via della Scienza 5, 09047 Selargius, Italy \\ ${ }^{7}$ Joint Institute for VLBI ERIC, Oude Hoogeveensedijk 4, 7991 PD Dwingeloo, The Netherlands \\ ${ }^{8}$ Sterrewacht Leiden, Leiden University, PO Box 9513, 2300 RA Leiden, The Netherlands
}

Received 17 August 2018 / Accepted 29 April 2019

\begin{abstract}
Context. The role of magnetic fields during the formation of high-mass stars is not yet fully understood, and the processes related to the early fragmentation and collapse are as yet largely unexplored. The high-mass star forming region G9.62+0.19 is a well known source, presenting several cores at different evolutionary stages.

Aims. We seek to investigate the magnetic field properties at the initial stages of massive star formation. We aim to determine the magnetic field morphology and strength in the high-mass star forming region G9.62+0.19 to investigate its relation to the evolutionary sequence of the cores.

Methods. We made use of Atacama Large Millimeter Array (ALMA) observations in full polarisation mode at $1 \mathrm{~mm}$ wavelength (Band 7) and we analysed the polarised dust emission. We estimated the magnetic field strength via the Davis-Chandrasekhar-Fermi and structure function methods.

Results. We resolve several protostellar cores embedded in a bright and dusty filamentary structure. The polarised emission is clearly detected in six regions: two in the northern field and four in the southern field. Moreover the magnetic field is orientated along the filament and appears perpendicular to the direction of the outflows. The polarisation vectors present ordered patterns and the cores showing polarised emission are less fragmented. We suggest an evolutionary sequence of the magnetic field, and the less evolved hot core exhibits a stronger magnetic field than the more evolved hot core. An average magnetic field strength of the order of $11 \mathrm{mG}$ was derived, from which we obtain a low turbulent-to-magnetic energy ratio, indicating that turbulence does not significantly contribute to the stability of the clump. We report a detection of linear polarisation from thermal line emission, probably from methanol or carbon dioxide, and we tentatively compared linear polarisation vectors from our observations with previous linearly polarised $\mathrm{OH}$ masers observations. We also compute the spectral index, column density, and mass for some of the cores.

Conclusions. The high magnetic field strength and smooth polarised emission indicate that the magnetic field could play an important role in the fragmentation and the collapse process in the star forming region G9.62+019 and that the evolution of the cores can be magnetically regulated. One core shows a very peculiar pattern in the polarisation vectors, which can indicate a compressed magnetic field. On average, the magnetic field derived by the linear polarised emission from dust, thermal lines, and masers is pointing in the same direction and has consistent strength.
\end{abstract}

Key words. stars: formation - ISM: magnetic fields - magnetic fields - polarization - stars: massive

\section{Introduction}

High-mass stars play a fundamental role in the evolution of the universe, but despite their importance, our comprehension of the physical mechanisms occurring in their formation is still incomplete. As a consequence, the high-mass star formation theory has been a topic of great debate in recent decades, and several models have been proposed such as the core accretion model (e.g. McKee \& Tan 2003; Banerjee \& Pudritz 2007) and the

\footnotetext{
${ }^{\star}$ A copy of the reduced images is available at the CDS via anonymous ftp to cdsarc.u-strasbg.fr $(130.79 .128 .5)$ or via http://cdsarc.u-strasbg.fr/viz-bin/qcat?J/A+A/626/A36
}

competitive accretion model (e.g. Bonnell \& Bate 2006). In particular, one of the subjects under discussion is the role played by the magnetic field at the first stages of massive star formation. While some theoretical and simulation works showed that magnetic fields can significantly influence each stage of massive star formation (e.g. Mouschovias \& Paleologou 1979; Mouschovias et al. 2006; Commerçon et al. 2011; Tan et al. 2013; Tassis et al. 2014; Klassen et al. 2017), other authors consider the effect of turbulence and gravity more important (e.g. Padoan \& Nordlund 2002; Klessen et al. 2005; Vázquez-Semadeni et al. 2011; Wang et al. 2014).

Recently, some theoretical simulations of molecular cloud collision show that massive star formation can be enhanced 
by the strong magnetic fields generated in the shocked region (Inoue et al. 2018; Inoue \& Fukui 2013; Vaidya et al. 2013). Moreover, other papers indicate that magnetic fields affect fragmentation, slow down cloud collapse, influence accretion, and drive feedback phenomena such as collimated outflows, which are important in removing excess angular momentum (McKee \& Ostriker 2007; Myers et al. 2013; Peters et al. 2014; Susa et al. 2015; Matsushita et al. 2018). These works indicate that turbulence can play a major role during the initial collapse in the regions where massive stars form, while magnetic fields are likely to be important close to the protostar, as evidenced by the detection of a pinched magnetic field in a few massive protostars (e.g. Girart et al. 2009; Qin et al. 2010). Furthermore, magnetic fields could also be required to stabilise discs and allow for the large accretion rate necessary during massive star formation (Johansen \& Levin 2008; Stepanovs et al. 2014). In addition, some authors suggest that non-ideal magnetohydrodynamic (MHD) effects such as ambipolar diffusion or Ohmic dissipation can explain some observed configurations such as accretion discs and outflows (Zhao et al. 2016; Machida et al. 2014; Seifried et al. 2012).

However it is still unclear which process dominates at which evolutionary stage, and more investigations are needed to overcome this uncertainty and to estimate the magnitude and morphology of the initial magnetic field.

A possible way to study the magnetic field parameters is through the analysis of dust polarisation observations. Interstellar dust produces thermal emission and extinction of light from a background star. The magnetic field morphology in the interstellar medium (ISM) can be probed by observing the linear polarisation of this radiation. This polarisation requires the alignment of irregularly shaped grains, as described by for example Cudlip et al. (1982); Hildebrand (1988); Draine \& Weingartner (1996, 1997); Lazarian (2000); Cho \& Lazarian (2005); Lazarian \& Hoang (2007, 2008); Hoang \& Lazarian (2008), and Andersson et al. (2015). The first successful observation of linearly polarised dust emission towards a molecular cloud was made by Hildebrand et al. (1984), and since then many other studies were presented (e.g. Crutcher 2012, and references therein). Interferometric images of the polarised emission of dust can show the magnetic field at a resolution of 100-1000 au (e.g. Girart et al. 2006; Attard et al. 2009; Houde et al. 2009; Hull et al. 2013; Zhang et al. 2014), and recently, thanks to the Atacama Large Millimeter Array (ALMA) capabilities, it is possible to trace magnetic fields close to the inner parts of the star forming cores (e.g. Girart et al. 2018; Alves et al. 2018; Maury et al. 2018; Beuther et al. 2018). However, this has been done only in a few projects, and we need more (multiwavelength) observations to draw a complete picture of the magnetic fields at different evolutionary stages of the protostar.

In this paper we investigate the magnetic field of the highmass star forming region G9.62+0.20 by analysing ALMA observations of its dust emission at $1 \mathrm{~mm}$ (band 7). We introduce G9.62+0.20 in Sect. 1. We describe our observations and the data reduction in Sect. 3. In Sect. 4 we present a high-resolution polarised image of the dust emission, and we show the morphology and the strength of the magnetic field. Then, we compare the results of the magnetic field direction strength obtained by our data with the previous results obtained by masers and we discuss this comparison in Sect. 5. In Sect. 6 we give our conclusions and future perspectives, considering that this single case of study can only be the starting point for a larger and more detailed study of magnetic fields.

\section{The case of $\mathrm{G} 9.62+0.20$}

G9.62+0.20 (hereafter G9.62) is a well-studied star forming region, located at a distance of $5.2 \mathrm{kpc}$ from the Sun (Sanna et al. 2009), which presents several cores at different evolutionary stages. This source contains a number of ultra-compact $\mathrm{H}$ II regions, various masers, and hot molecular cores which drive molecular outflows (e.g. Persi et al. 2003; Linz et al. 2005; Liu et al. 2017, hereafter L17 and references therein). The filament shows a fairly well-established evolutionary sequence, making it clear that a sequential high-mass star formation is occurring in the G9.62 complex on a parsec-scale, from west to east (Testi et al. 1998, 2000; Liu et al. 2011).

Figure 1 presents a Spitzer/IRAC three-colour composite image of the region at $8,4.5$, and $3.6 \mu \mathrm{m}$ (in red, green, and blue, respectively). In this figure, letters from A to I denote the massive young stellar objects that are known centimetre and millimetre sources. The dark blue dots indicate the positions of the evolved young stellar objects $\mathrm{A}, \mathrm{B}$, and $\mathrm{C}$, while orange dots shows the less evolved objects. As shown in Fig. 1, the youngest sources are located in a dense molecular clump to the east of A, B, and C (Garay et al. 1993).

The L17 authors estimated for the G9.62 clump a mass of $\sim 2800 \pm 200 M_{\odot}$, a luminosity of $\sim(1.7 \pm 0.1) \times 10^{6} L_{\odot}$ and a mean number density of $\sim(9.1 \pm 0.7) \times 10^{4} \mathrm{~cm}^{-3}$. Observations by JCMT/SCUBA at $450 \mu \mathrm{m}$ also traced the dust emission from the new generation of massive protostars which are forming inside the clump and which are surrounded by photodissociation regions (PDRs) mapped by Spitzer/IRAC observations of polycyclic aromatic hydrocarbon $(\mathrm{PAH})$ at $8 \mu \mathrm{m}$ (red contours in Fig. 1) (Hofner et al. 2001; Testi et al. 2000; Liu et al. 2011).

Submillimeter Array (SMA) observations at $345 \mathrm{GHz}$ $(860 \mu \mathrm{m})$ clearly revealed multiple components (Liu et al. 2011), as shown in Fig. 1 with yellow contours. The strongest $\left(\sim 1 \mathrm{Jy} \mathrm{beam}^{-1}, \sim 4.7^{\prime \prime} \times 1.3^{\prime \prime}\right)$ is concentrated on G9.62+0.20F. This component, with no detectable H II region but still surrounded by hot dust, is in an early phase and presents signatures of disc accretion (Cesaroni et al. 1994) and it should eventually evolve into a B0 star (Testi et al. 1998). The SMA revealed a second component $\left(\sim 0.8 \mathrm{Jy}\right.$ beam $\left.^{-1}, \sim 1.5^{\prime \prime} \times 1.3^{\prime \prime}\right)$ that is concentrated on region $\mathrm{E}$. The more evolved UC $\mathrm{H}$ II region G9.62+0.20E, likely excited by a B1 star, hosts the strongest 6.7 GHz methanol maser known (Vlemmings 2008; Fish et al. 2005b). The same SMA observations also detected a smaller third core, located in the north-west part of the filament closer to G9.62+0.20C ( 0.2 Jy beam ${ }^{-1}$, unresolved with SMA, $\sim 1.2^{\prime \prime}$ in size) with no other related star formation tracers. This could thus be a failed core, where the gravity potential was unable to overcome the magnetic pressure or a remnant core in the envelope of the UC H II region G9.62+0.20C (Liu et al. 2011).

Recent ALMA observations at $230 \mathrm{GHz}$ further resolved the G9.62 clump into 12 dense cores (named MM1-MM12, L17), presenting a range of mass spanning from $4 M_{\odot}$ to $87 M_{\odot}$. Five of the 12 cores are manifest massive protostars. In L17, a possible evolutionary sequence was proposed by the analysis of the chemistry and the outflow morphology detected for each core, which is discussed in Sect. 5. In this paper, we refer to the entire core $n$ as MM $n$ (e.g. MM8) and to its subcores by adding a letter to the core name (e.g. MM8a, MM8b).

\section{Observations and data reduction}

G9.62 was observed with ALMA in band 7 between 13 May for $\sim 3.5 \mathrm{~h}$ and 27 June 2016 for $\sim 4.5 \mathrm{~h}$ (Project 2015.1.01349.S, 


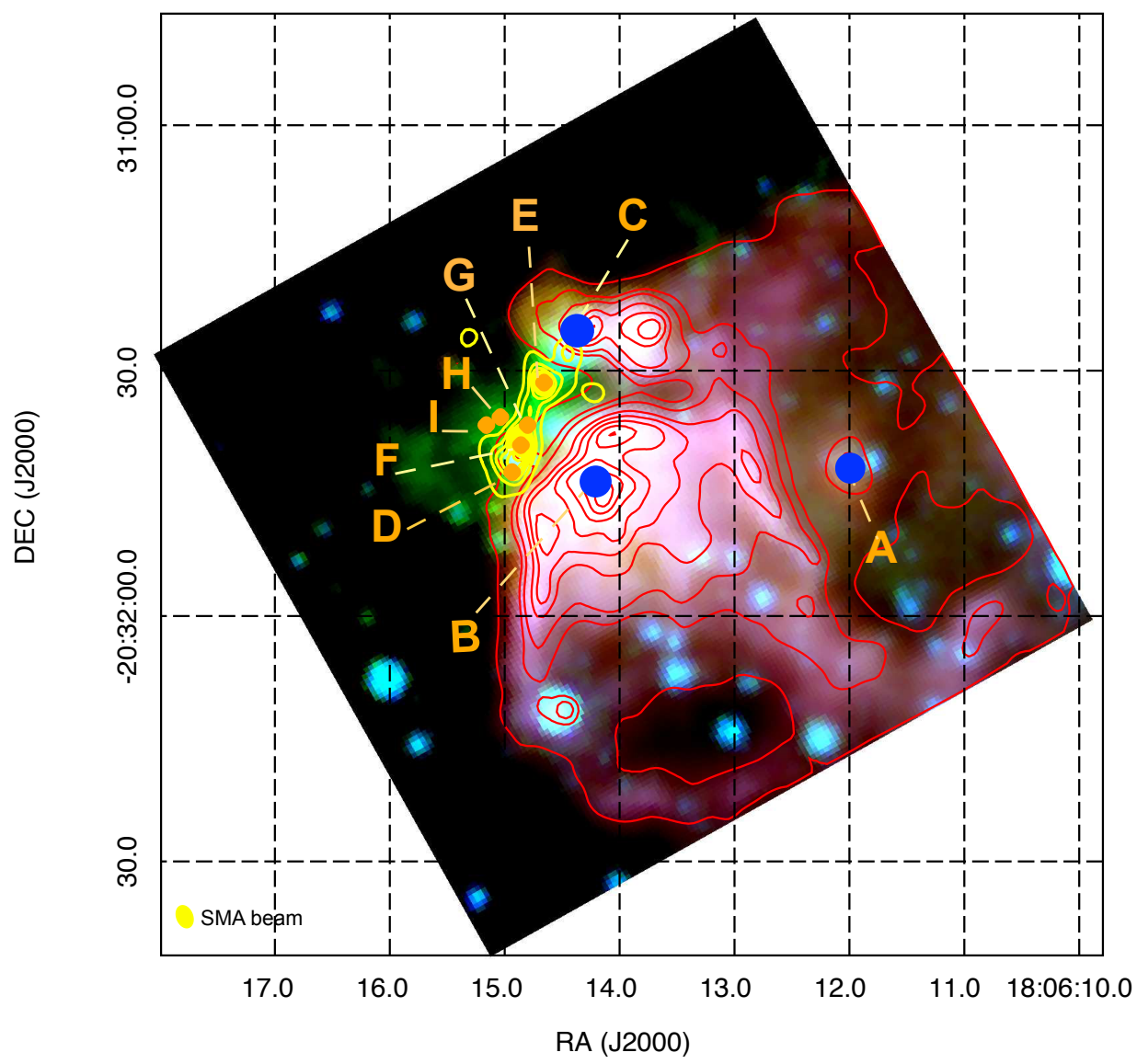

Fig. 1. Spitzer/IRAC three-colour composite image at $8,4.5$, and $3.6 \mu \mathrm{m}$ in red, green, and blue, respectively, of the star forming region G9.62. Red contours maps the Spitzer/IRAC observations of PAH at $8 \mu \mathrm{m}$. The contours level are linearly from $(0.1-0.9) \times$ $2550 \mathrm{mJy} \mathrm{sr}^{-1}$. The dark blue dots indicate the position of the more evolved source A, $\mathrm{B}$, and $\mathrm{C}$. The orange dots denote the positions of the massive young stellar objects denoted by the letters from D to I. Yellow contours track the continuum emission from $860 \mu \mathrm{m}$ SMA observations of the G9.62 clump (Liu et al. 2011). The contours are drawn from $0.03 \mathrm{Jy}^{-1}$ beam ${ }^{-1}$ and rising in steps of $0.12 \mathrm{Jy} \mathrm{beam}^{-1}$. The noise level is $0.01 \mathrm{Jy}^{-1}$ beam ${ }^{-1}$ Liu et al. 2011).
PI: Wouter Vlemmings). The observations were performed using 39 antennas of the ALMA $12 \mathrm{~m}$ array in May and 42 antennas in June.

The four spectral windows with $2 \mathrm{GHz}$ bandwidth each were centred at $336.5,338.5,348.5$, and $350.5 \mathrm{GHz}$ and were divided into 64 channels. The channel width is $31.25 \mathrm{MHz}$. At our frequency, the primary beam of the ALMA $12 \mathrm{~m}$ array is $20^{\prime \prime}$, which is large enough to encompass all our region of interest. However, since the ALMA observatory currently regards only the inner one-third of the primary beam as reliable for polarisation measurements, we used two fields centred on the main continuum sources.

The phase centres of the two fields are (at J2000) RA 18:06:14.558, Dec -20.31.30.05 and RA 18:06:14.889, Dec -20.31 .40 .149 . The two fields are named North field and South field. Two different array configurations were used (C36$3, \mathrm{C} 36-4)$ with a total baseline range between 17 and $630 \mathrm{~m}$ in May and between 15 and $850 \mathrm{~m}$ in June ${ }^{1}$. The two array configurations correspond to a maximum recoverable scale (MRS) $\theta_{\text {MRS }} \sim 2.8^{\prime \prime}$.

The datasets were reduced using the Common Astronomy Software Application package (CASA version 4.6.0), and the calibration was performed using J1924-2914 and J1751+0939 as bandpass calibrator, J1733-1304 as flux calibrator, and J18322039 as phase calibrator. J1924-2914 was also used as polarisation calibrator. For the calibration we followed the procedures provided by the ALMA observatory. One spectral window was partially flagged before imaging due to contamination by molecular lines (see Sect. 4). The synthesised beam of the image is

\footnotetext{
1 http://almascience.eso.org/observing/prior-cycleobserving-and-configuration-schedule
}

$0.34^{\prime \prime} \times 0.25^{\prime \prime}\left(\mathrm{PA} 88.53^{\circ}\right)$. The $1 \sigma \mathrm{rms}$ value of the Stokes I is $0.15 \mathrm{mJy}$ beam $^{-1}$, reaching a dynamic range of 294 . The continuum image suffers dynamic range limitation because of partially resolved out flux. Comparing the flux of the two brighter cores $\mathrm{E}$ and $\mathrm{F}$ from previous SMA observations (Liu et al. 2011), for which the flux is extracted over a similar region in our ALMA observations, we estimate a flux loss between 30-60\%, respectively, on a scale larger than $\theta_{\text {MRS }}$. The absolute flux loss of our entire region compared to single dish observations (obtained from the ATLASGAL database Thompson et al. 2006) over the same area is of the order of $70 \%$.

For the polarisation calibration, we followed the procedures provided by the ALMA observatory. The minimum polarisation value in our data is higher than the minimum value recommended by ALMA $(0.3 \%$ of the total intensity of each core, see Tables 1 and 2) and any remaining instrumental polarisation signatures are smaller than $0.1 \mathrm{mJy}^{\text {beam }}{ }^{-1}$. The analysis of the linear polarisation was conducted on each target field imaged separately, and considering only the main substructures inside the recommended inner third of the primary beam for the two pointings, as shown in Fig. 2. The $1 \sigma$ rms value of the linear polarised image is on average $\sigma_{\mathrm{P}}=0.08 \mathrm{mJy}$ beam $^{-1}$, obtained using $\sigma_{\mathrm{P}}=\sqrt{\left[\left(Q \times \sigma_{Q}\right)^{2}+\left(U \times \sigma_{U}\right)^{2}\right] /\left(Q^{2}+U^{2}\right)}$ and we conducted the polarisation analysis above a signal-to-noise ratio $(\mathrm{S} / \mathrm{N})$ of 4 . This selection was applied to perform the analysis of the polarised emission and the magnetic field only in the proximity of the cores. We combined the $U$ and $Q$ data cubes to produce cubes of polarised intensity $\left(I_{\mathrm{P}}=\sqrt{Q^{2}+U^{2}-\sigma_{\mathrm{P}}^{2}}\right)$ and polarisation angle $(\psi=1 / 2 \times \operatorname{atan}(U / Q))$. The error on polarisation angles includes the formal error due to the thermal noise (Wardle \& Kronberg 1974) and this error is given 
A\&A 626, A36 (2019)

Table 1. Parameters of the continuum sources of the $1 \mathrm{~mm}$ ALMA observations of G9.62+0.19.

\begin{tabular}{|c|c|c|c|c|c|c|c|c|c|c|c|c|c|}
\hline Core & & $\begin{array}{c}\mathrm{RA}^{(a)} \\
\text { Offset }\left({ }^{\prime \prime}\right)\end{array}$ & $\begin{array}{c}\operatorname{Dec}(a) \\
\text { Offset }\left({ }^{\prime \prime}\right)\end{array}$ & $\begin{array}{c}I_{\text {Peak }}{ }^{(b)} \\
\left(\mathrm{mJy}^{-1} \text { beam }^{-1}\right)\end{array}$ & $\begin{array}{c}\text { Integrated flux } \\
(\mathrm{mJy})\end{array}$ & $\begin{array}{c}a^{(d)} \\
\text { (milliarcsec) }\end{array}$ & $\begin{array}{c}b^{(d)} \\
\text { (milliarcsec) }\end{array}$ & $\mathrm{PA}^{(d)}$ & $\alpha^{(e)}$ & $\beta^{(f)}$ & $\begin{array}{c}N_{\mathrm{H}_{2}}(f) \\
\mathrm{cm}^{-2}\end{array}$ & $\begin{array}{c}\text { Mass } \\
M_{\odot}\end{array}$ & $\lambda^{(g)}$ \\
\hline MM1a & & $-4.58 \pm 0.04$ & $7.56 \pm 0.02$ & $24.5 \pm 2.7$ & $114.0 \pm 13.0$ & $726 \pm 4$ & $426 \pm 7$ & $110.6 \pm 9.6$ & $3.4 \pm 0.5$ & 1.6 & $2.9 \times 10^{24}$ & 10 & - \\
\hline MM1b & & $-3.93 \pm 0.05$ & $6.75 \pm 0.02$ & $21.4 \pm 2.7$ & $98.0 \pm 12.0$ & $712 \pm 2$ & $432 \pm 3$ & $90 \pm 12$ & - & - & - & - & - \\
\hline MM2 & & $-4.06 \pm 0.00$ & $4.46 \pm 0.00$ & $11.0 \pm 0.0$ & $19.2 \pm 0.0$ & $374 \pm 13$ & $138 \pm 42$ & $117 \pm 16$ & - & - & - & - & - \\
\hline MM3a & & $-0.20 \pm 0.00$ & $5.06 \pm 0.00$ & $27.6 \pm 2.0$ & $221.0 \pm 18.0$ & $1251 \pm 105$ & $419 \pm 47$ & $0.06 \pm 0.02$ & $3.3 \pm 0.3$ & 1.5 & $3.5 \times 10^{24}$ & 21 & 8 \\
\hline MM3b & & $-0.25 \pm 0.00$ & $4.02 \pm 0.00$ & $23.0 \pm 2.7$ & $54.4 \pm 8.8$ & $384 \pm 69$ & $281 \pm 93$ & $1.16 \pm 0.68$ & - & - & - & - & - \\
\hline MM4a & $\mathrm{E}$ & $-1.53 \pm 0.00$ & $3.35 \pm 0.00$ & $321.6 \pm 6.8$ & $699.0 \pm 20.0$ & $402 \pm 18$ & $238 \pm 15$ & $58.4 \pm 4.5$ & $3.8 \pm 0.2$ & 1.8 & $9.5 \times 10^{24}$ & 43 & 6 \\
\hline MM4b & $\mathrm{E}$ & $-1.84 \pm 0.03$ & $3.25 \pm 0.02$ & $82.6 \pm 5.3$ & $508.0 \pm 37.0$ & $1162 \pm 94$ & $345 \pm 34$ & $119.4 \pm 2.1$ & - & - & - & - & - \\
\hline MM5 & & $-1.19 \pm 0.02$ & $2.20 \pm 0.01$ & $43.1 \pm 6.2$ & $42.4 \pm 6.1$ & - & - & - & - & - & - & - & - \\
\hline MM6a & & $-0.04 \pm 0.00$ & $0.13 \pm 0.00$ & $65.6 \pm 1.9$ & $64.6 \pm 1.8$ & - & - & - & - & - & - & - & - \\
\hline MM6b & & $0.40 \pm 0.02$ & $-0.37 \pm 0.02$ & $14.8 \pm 1.9$ & $14.6 \pm 1.8$ & - & - & - & - & - & - & - & - \\
\hline MM6c & & $0.06 \pm 0.01$ & $-0.01 \pm 0.02$ & $42.6 \pm 1.6$ & $251.0 \pm 11.0$ & $1057 \pm 50$ & $324 \pm 24$ & $167.3 \pm 1.5$ & $3.9 \pm 0.3$ & 2.1 & $5.8 \times 10^{24}$ & 22 & - \\
\hline MM7 & $\mathrm{G}$ & $0.30 \pm 0.01$ & $-2.30 \pm 0.01$ & $168.9 \pm 11.0$ & $321.0 \pm 30.0$ & $359 \pm 45$ & $152 \pm 82$ & $175 \pm 15$ & $3.8 \pm 0.5$ & 1.8 & $3.5 \times 10^{25}$ & 15 & - \\
\hline MM8a & $\mathrm{F}$ & $1.32 \pm 0.00$ & $-4.52 \pm 0.00$ & $366.7 \pm 7.5$ & $675.0 \pm 20.0$ & $342 \pm 17$ & $210 \pm 11$ & $85.2 \pm 4.1$ & $3.8 \pm 0.3$ & 1.8 & $4.7 \times 10^{25}$ & 38 & 21 \\
\hline MM8b & $\mathrm{F}$ & $1.15 \pm 0.02$ & $-4.73 \pm 0.04$ & $80.7 \pm 4.8$ & $1011.0 \pm 65.0$ & $1612 \pm 107$ & $568 \pm 44$ & $7.2 \pm 2.2$ & - & - & - & - & - \\
\hline MM8c & $\mathrm{F}$ & $1.25 \pm 0.03$ & $-3.75 \pm 0.01$ & $44.6 \pm 6.9$ & $43.9 \pm 6.8$ & - & - & - & - & - & - & - & - \\
\hline MM9 & & $3.51 \pm 0.03$ & $-6.43 \pm 0.02$ & $63.4 \pm 7.6$ & $147.0 \pm 17.0$ & $364 \pm 0$ & $310 \pm 0$ & $87.8 \pm 0.1$ & $3.1 \pm 0.7$ & 1.3 & $1.9 \times 10^{25}$ & 23 & - \\
\hline MM10 & & $2.18 \pm 0.04$ & $-6.61 \pm 0.03$ & $23.0 \pm 4.1$ & $53.4 \pm 9.6$ & $402 \pm 34$ & $259 \pm 47$ & $36 \pm 36$ & - & - & - & - & - \\
\hline MM11a & D & $2.03 \pm 0.01$ & $-8.26 \pm 0.03$ & $42.8 \pm 2.1$ & $277.0 \pm 15.0$ & $1046 \pm 62$ & $363 \pm 32$ & $172.1 \pm 2.1$ & $2.7 \pm 0.4$ & 0.9 & $8.7 \times 10^{24}$ & 37 & - \\
\hline MM11b & D & $2.30 \pm 0.02$ & $-8.19 \pm 0.01$ & $20.3 \pm 2.4$ & $20.0 \pm 2.4$ & - & - & - & - & - & - & - & - \\
\hline MM11c & D & $1.91 \pm 0.01$ & $-8.78 \pm 0.02$ & $17.3 \pm 2.4$ & $17.1 \pm 2.4$ & - & - & - & - & - & - & - & - \\
\hline MM12a & & $3.20 \pm 0.03$ & $-10.81 \pm 0.03$ & $6.8 \pm 1.4$ & $8.7 \pm 1.8$ & - & - & - & - & - & - & - & - \\
\hline MM12b & & $3.42 \pm 0.03$ & $-11.38 \pm 0.04$ & $6.2 \pm 1.4$ & $8.3 \pm 1.9$ & - & - & - & - & - & - & - & - \\
\hline MM12c & & $3.68 \pm 0.03$ & $-12.16 \pm 0.03$ & $7.3 \pm 1.4$ & $10.3 \pm 2.0$ & - & - & - & - & - & - & - & - \\
\hline
\end{tabular}

Notes. ${ }^{(a)}$ The offsets are relative to the absolute position $\alpha_{2000}=18^{\mathrm{h}} 06^{\mathrm{m}} 14.78^{\mathrm{s}} \delta_{2000}=-20^{\circ} 31^{\prime} 34.9^{\prime \prime} .{ }^{\left({ }^{b}\right)}$ Peak flux density. ${ }^{(c)}$ Total flux density from the 2D Gaussian fit. To model MM6, MM8, and MM11 we make use of Gaussian components and point-like source components. CASA task IMFIT was used to perform the Gaussian fit. ${ }^{(d)} a, b$, and PA are the deconvolved FWHM major and minor axes and position angle, obtained from 2D Gaussian fits. Point sources are those for which $a, b$, and PA are missing. Uncertainties of 0.00 and 0.0 are $<0.01$ and $<0.1$, respectively. ${ }^{(e)}$ Spectral index $\alpha$ obtained between our ALMA band 7 observations and ALMA band 6 observations by Liu et al. 2017. ${ }^{(f)}$ The spectral index of the dust opacity $\beta$, the column density $N_{\mathrm{H}_{2}}$, and the mass in solar units are estimated considering a temperature of the dust $T_{\mathrm{d}} \sim 35 \mathrm{~K}$ for the starless core MM1a, MM3a, MM9, and for the young sources MM6c and MM11a, while a $T_{\mathrm{d}} \sim 100 \mathrm{~K}$ was used for the hot cores MM4a, MM7 and MM8a. ${ }^{(g)}$ To compute the mass to flux ratio we used the magnetic field values obtained by SF analysis.

Table 2. Polarised intensities and magnetic field parameters.

\begin{tabular}{lcccccccc}
\hline \hline Core & $\begin{array}{c}I_{\text {peak }} \\
\left(\mathrm{mJy} \mathrm{beam}^{-1}\right)\end{array}$ & $\begin{array}{c}I_{\text {pol }}{ }^{(a)} \\
\left(\mathrm{mJy} \mathrm{beam}^{-1}\right)\end{array}$ & $\begin{array}{c}\sigma_{\psi}{ }^{(b)} \\
\left({ }^{\circ}\right)\end{array}$ & $\begin{array}{c}\sigma_{v}{ }^{(c)} \\
\left(\mathrm{km} \mathrm{s}^{-1}\right)\end{array}$ & $\begin{array}{c}B_{\perp}^{\mathrm{DCF}} \\
(\mathrm{mG})\end{array}$ & $\begin{array}{c}b \\
\left({ }^{\circ}\right)\end{array}$ & $\begin{array}{c}B_{\perp}^{\mathrm{SF}} \\
(\mathrm{mG})\end{array}$ & $B_{\mathrm{t}} / B_{0}$ \\
\hline MM3 & 27.6 & $2.79 \pm 0.07$ & $41.8 \pm 0.1$ & $2.00 \pm 0.01$ & $0.33 \pm 0.02$ & $11.9 \pm 0.2$ & $3.30 \pm 0.07$ & 0.2 \\
MM4 & 321.6 & $1.80 \pm 0.06$ & $28.0 \pm 0.4$ & $2.00 \pm 0.01$ & $1.57 \pm 0.02$ & $9.4 \pm 0.3$ & $13.18 \pm 0.42$ & 0.1 \\
MM7 & 168.9 & $0.66 \pm 0.08$ & - & - & - & - & - & - \\
MM8a & 366.7 & $1.74 \pm 0.08$ & $30.5 \pm 3.8$ & $2.35 \pm 0.01$ & $1.64 \pm 0.02$ & $8.6 \pm 0.4$ & $17.10 \pm 0.75$ & 0.1 \\
MM8c & 44.6 & $1.25 \pm 0.08$ & - & - & - & - & - & - \\
MM9 & 63.4 & $0.91 \pm 0.07$ & - & - & - & - & - & - \\
MM11a & 42.8 & $0.54 \pm 0.10$ & - & - & - & - & - & - \\
\hline
\end{tabular}

Notes. ${ }^{(a)} I_{\text {pol }}$ are taken at the peak of the polarised intensity. In case of MM3 and MM4 the peak is located in MM3a and MM4a, respectively. ${ }^{(b)}$ All the values of $\sigma_{\psi}$ are obtained using the classical variance, calculated around the circular mean (Jammalamadaka \& SenGupta 2001; Pewsey et al. 2013), over regions covering MM3, MM4, and MM8. ${ }^{(c)}$ All the values of $\sigma_{v}$ are obtained by Liu et al. 2017, Table 3, using methanol emissions.

by $\sigma_{\text {tn }}=0.5\left(\sigma_{\mathrm{P}} / \mathrm{I}_{\mathrm{P}}\right) \times\left(180^{\circ} / \pi\right)$. For the $4 \sigma$ cut this value corresponds to $7.2^{\circ}$ and for the strongest peaks this decreases to $0.7^{\circ}$.

\section{Results}

\subsection{Detection of the cores}

In Fig. 2 we present the Stokes I image of the G9.62 clump as seen in our ALMA band 7 continuum observations. The two yellow circles outline the inner third of the two pointings that we observed in this project. The filament presents a highly fragmented structure, extending from the north-west to the south-east direction. We confirm the presence of the 12 dense cores, including the main three continuum peaks MM3, MM4, and MM8, already seen in the previous ALMA band 6 observations by L17. In addition, since our ALMA band 7 observations have about three times higher angular resolution than L17, we identify several other substructures that have $\mathrm{S} / \mathrm{Ns}$ above $40 \sigma$. In Table 1 we list 23 cores and their characteristics, and we identify these in Fig. 3. The positions, peak flux densities, integrated fluxes, and sizes of the cores are obtained from two-dimensional Gaussian 


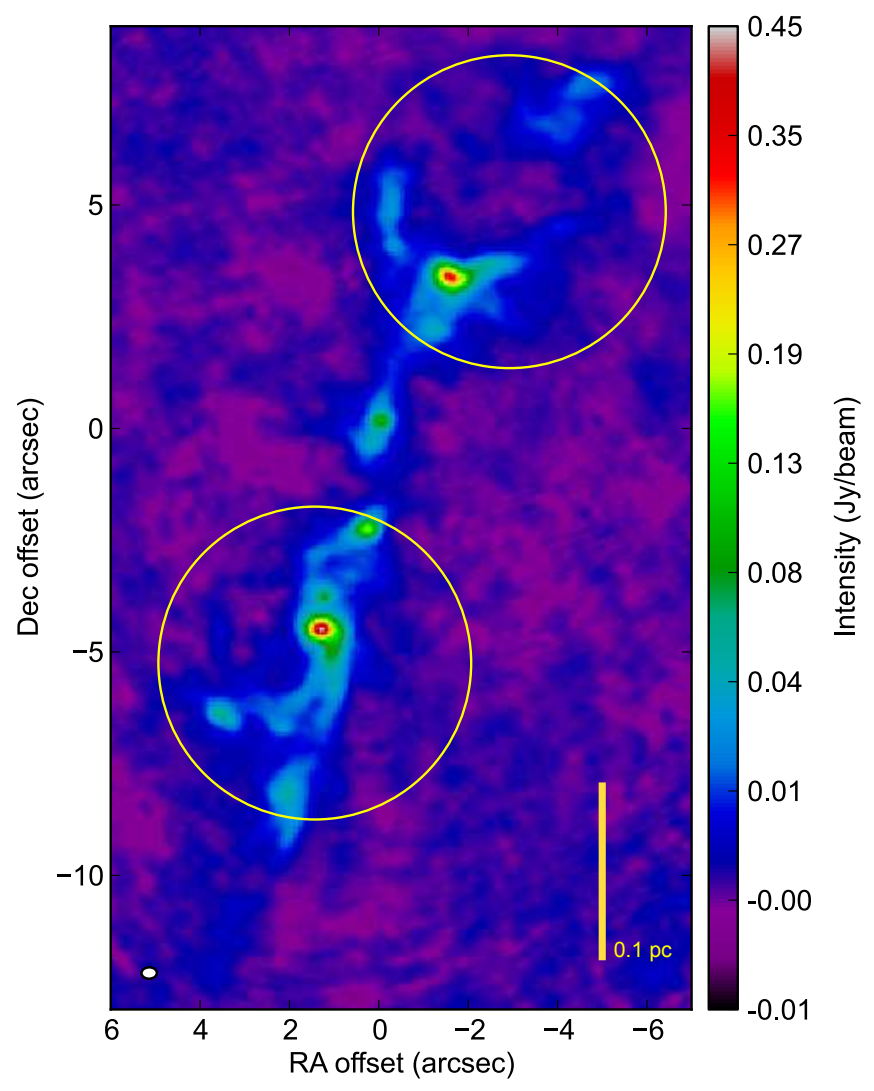

Fig. 2. Total intensity image of the star forming region G9.62+0.19 at $1 \mathrm{~mm}$ wavelength. The yellow circles with the diameter of $\sim 7^{\prime \prime}$ indicate the inner one-third of the primary beam for the two pointings which are overlapping. The yellow bar indicates the physical scale of $0.1 \mathrm{pc}$, computed at the distance of the source. The colour bar indicates the intensity of the emission in $\mathrm{Jy}$ beam $^{-1}$. The offsets are relative to the absolute position $\alpha_{2000}=18^{\mathrm{h}} 06^{\mathrm{m}} 14.78000^{\mathrm{s}}, \delta_{2000}=-20^{\circ} 31^{\prime} 34.90^{\prime \prime}$. The white ellipse represents the beam.

fits performed with CASA task IMFIT. We observe the presence of large amount of extended emission that we model using single Gaussian and point-like sources. This analysis is based on the combined dataset of both observed fields.

\subsection{Spectral index of the dust emission}

In Table 1 we also give the values of the spectral index $\alpha$, computed considering the integrated intensities of the cores from ALMA band 6 observations (L17) and similar visibility coverage to our observations. For a relevant measurement of the spectral index, each of the spectral windows in the two datasets were imaged (line free channels) using only the overlapping $u v$ distances (20-360 $\mathrm{k} \lambda$ ) and restoring the images with a circular beam of $0.9^{\prime \prime}$. While this limits the comparison to the peaks given in L17, it can hint at the evolutionary status of the cores which can then be compared to the polarisation images.

The dust emission at submillimetre wavelengths can be represented by a grey body, with intensity varying smoothly as a function of frequency. Having measured the intensity of dust emission at two submillimetre frequencies, it is possible to describe the spectral energy distribution (SED) of each core using the spectral index of the dust emission $\alpha$ defined as

$\alpha=\frac{\ln \left(S_{\mathrm{a}} / S_{\mathrm{b}}\right)}{\ln \left(v_{\mathrm{b}} / v_{\mathrm{a}}\right)}$, where $S_{\mathrm{a}}$ and $S_{\mathrm{b}}$ are flux densities at frequencies $v_{\mathrm{a}}$ and $v_{\mathrm{b}}$, respectively (Williams et al. 2004).

Within the errors all the cores present roughly the same spectral index. Since $\alpha$ depends on a combination of several factors, the uncertainties are influenced by the errors on the opacity, beam-averaged dust temperature, and spectral index of the dust opacity $\left(\beta\right.$, defined as $\left.\tau \propto v^{\beta}\right)$. Also the absolute flux calibration uncertainty is a significant source of error, although this is a systematic error in the spectral index for all regions that can be up to 0.2 (based on $10 \%$ flux calibration uncertainties). Moreover, changes in the composition of the dust grains and temperature variations through the dust envelope can influence $\alpha$. The models developed by Ossenkopf \& Henning (1994) predicted that $\beta$ changes if the dust grains do not have ice mantles; this should be the case of some of our cores, since they are in the hot core phase reaching inner dust temperatures higher than $100 \mathrm{~K}$. This fact is also confirmed by the detection of $\mathrm{CH}_{3} \mathrm{OH}$ and $\mathrm{CH}_{3} \mathrm{CN}$, well-known molecular tracers of hot cores (Qiu et al. 2014), pointing to high temperature and high density regions where the environment is warm enough to melt ice mantles and permit grain growth (Williams et al. 2004).

We can use an estimate of the temperature of dust grains within the cores to calculate the spectral index of the dust opacity

$\beta=(\alpha+\Delta \alpha)-2$

where $\Delta \alpha$ is defined as the Rayleigh-Jeans correction factor (Williams et al. 2004).

The dust temperatures are closer to the equivalent temperature at a given frequency $T_{v}=h v / k$ than a Rayleigh-Jeans approximation would permit, where $h$ is the Planck constant and $k$ is the Boltzmann constant in cgs unit. Thus, for frequencies $v_{\mathrm{a}}=230 \mathrm{GHz}$ and $v_{\mathrm{b}}=340 \mathrm{GHz}$ in ALMA band 6 and band 7, we obtain $T_{v_{\mathrm{a}}}=11 \mathrm{~K}$ and $T_{v_{\mathrm{b}}}=16 \mathrm{~K}$, respectively, from which we can estimate the correction $\Delta \alpha$ as

$\Delta \alpha=\frac{\ln \left(\frac{e^{T_{v_{\mathrm{b}}} / T_{\mathrm{d}}}-1}{e^{T_{v a} / T_{\mathrm{d}}-1}}\right)}{\ln \left(T_{v_{\mathrm{b}}} / T_{v_{\mathrm{a}}}\right)}-1$.

Assuming a dust temperature of $T_{\mathrm{d} 1} \sim 100 \mathrm{~K}$ and $T_{\mathrm{d} 2} \sim 35 \mathrm{~K}$ in the hot cores and in the starless cores, respectively (values proposed by L17), we obtain from Eq. (3) $\Delta \alpha_{1}=0.06$ and $\Delta \alpha_{2}=0.20$ in the two environments. We then estimate the spectral index of the dust opacity $\beta$ using Eq. (2), and report all the values in Table 1 . Since the error on $\Delta \alpha$ depends on the uncertainty on $T_{\mathrm{d}}$, which were not estimated by L17, the error on $\beta$ has the same order of uncertainties of the spectral index $\alpha$ and could be underestimated.

\subsection{Column densities and masses of the cores}

Given the above values for the dust temperature $T_{\mathrm{d}}$, and following the approach described in L17, we can estimate the $\mathrm{H}_{2}$ column densities $\left(N_{\mathrm{H}_{2}}\right)$ and gas masses for the regions. The integrated flux $S_{v}$ from thermal dust is

$S_{v}=B_{v}\left(T_{\mathrm{d}}\right)\left[1-e^{-\tau_{\mathrm{d}}}\right] \Omega_{\mathrm{s}}$,

where the observed frequency $v$ is expressed in $\mathrm{Hz}, \Omega_{\mathrm{s}}$ is the solid angle in steradiants, and $\tau_{\mathrm{d}}$ is the optical depth. From the Planck function

$B_{v}\left(T_{\mathrm{d}}\right)=\frac{2 h v^{3}}{c^{2}} \frac{1}{e^{\frac{h v}{k T_{\mathrm{d}}}}-1}$, 

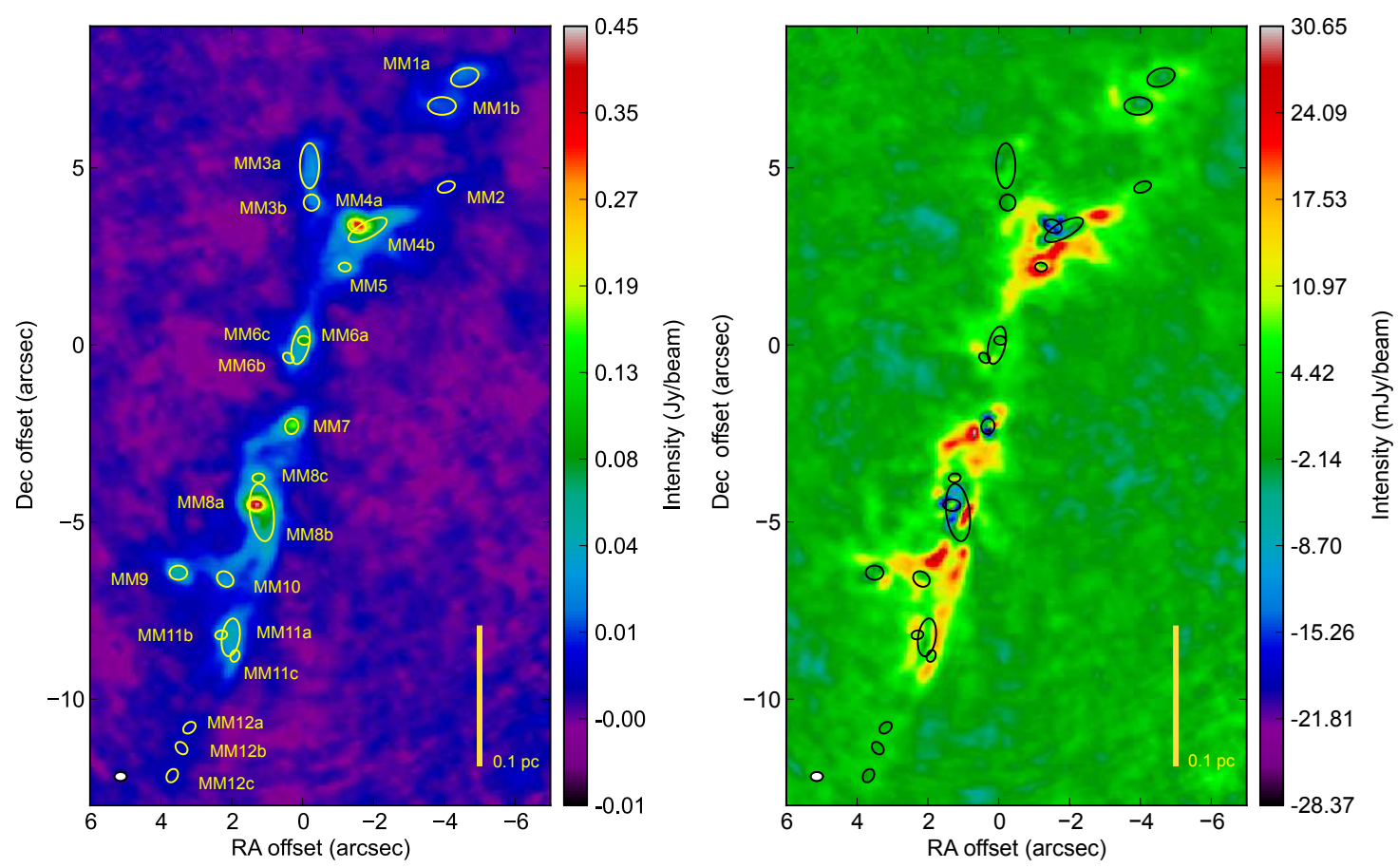

Fig. 3. Left panel: total intensity image of the star forming region G9.62+0.19, with the identified cores. The offsets are relative to the absolute position $\alpha_{2000}=18^{\mathrm{h}} 06^{\mathrm{m}} 14.78000^{\mathrm{s}}, \delta_{2000}=-20^{\circ} 31^{\prime} 34.90^{\prime \prime}$. The white ellipse represents the beam and the yellow ellipses represent the dense cores identified by the Gaussian fit and illustrated in Table 1 . The colour scale goes from -0.01 to $0.45 \mathrm{Jy} \mathrm{beam}^{-1}$. Right panel: residual image in which the black ellipses represent the dense cores as before. The colour scale goes from -28.37 to $30.65 \mathrm{mJy} \mathrm{beam}^{-1}$. In both panels, the yellow bar indicates the physical scale of $0.1 \mathrm{pc}$, at the distance of the source.

where $\mathrm{c}$ is the velocity of light in $\mathrm{cm} \mathrm{s}^{-1}$. Considering a dust temperature of $T_{\mathrm{d} 1} \sim 100 \mathrm{~K}$ and $T_{\mathrm{d} 2} \sim 35 \mathrm{~K}$ in the hot cores and in the starless cores, we can derive from Eq. (5) the optical depth $\tau_{d}$ and we can compute the mass of the dust $M_{\mathrm{d}}$, which is defined as

$M_{\mathrm{d}}=\frac{\Omega_{\mathrm{s}} d^{2} \tau_{\mathrm{d}}}{\kappa_{v}}$

where $\kappa_{v}$ is the dust opacity per unit dust mass and $d$ is the distance in $\mathrm{cm}$. We derived the dust opacity index $\kappa_{v}$ from Ossenkopf \& Henning (1994) for grains with ice mantles using

$\kappa_{v}=\kappa_{0}\left(\frac{v}{v_{0}}\right)^{\beta}$.

We also take into account two different density regimes for dense hot cores and for starless cores, as reported in L17. In the first case we adopted an average number density $n=10^{6} \mathrm{~cm}^{-3}$ for the denser cores MM4, MM7, and MM8 and $\kappa_{300 \mathrm{GHz}} \sim$ $1.37 \mathrm{~cm}^{2} \mathrm{~g}^{-1}$ provided by Ossenkopf \& Henning (1994). In the second case, we assumed $n=10^{5} \mathrm{~cm}^{-3}$ for starless cores MM1a, MM3a, MM9, and for the young sources MM6c and MM11a and we derived $\kappa_{v}$ from $\kappa_{230 \mathrm{GHz}} \sim 0.9 \mathrm{~cm}^{2} \mathrm{~g}^{-1}$ given by Ossenkopf \& Henning (1994). We consider a gas-to-dust mass ratio of 100 and thus we obtained the column densities from

$N_{\mathrm{H}_{2}}=\frac{M_{\mathrm{g}}}{\tau_{d}} \mu \mathrm{m}_{\mathrm{H}} \Omega_{\mathrm{s}} d^{2}$,

where $M_{\mathrm{g}}$ is the mass of the gas.

The derived values are presented in Table 1: the core masses range between roughly $10 M_{\odot}$ and $40 M_{\odot}$ and the column densities between $2 \times 10^{24}$ and $6 \times 10^{25} \mathrm{~cm}^{2}$, which are a factor of
10-100 larger than the mean values reported in L17. The optical depth ranges typically between 0.2 and 0.9 with uncertainties of $\sim 20 \%$, mainly determined by the errors on the dust model and on the radii from the Gaussian fits, spectral index, and temperature. For sources MM7 and MM8 the errors on the optical depth are much larger and we can only constrain $\tau_{\mathrm{d}}<3.5$. The uncertainties on the optical depth may cause an overestimation of the mass if the emission becomes optically thick. Therefore considering all these uncertainties, the errors of the masses and column densities are difficult to quantify and they could be more than a factor of 5 and less than a factor of 10 .

\subsection{Detection of linear polarisation and magnetic field morphology}

We detect linear polarised emission in six regions: two in the northern field (from MM3 and MM4) and four in the southern field (from MM7, MM8, MM9 and MM11a). The total intensity $\left(I_{\text {peak }}\right)$ and linear polarised intensity $\left(I_{\text {pol }}\right)$ are reported in Table 2. The linear polarised emission coming from the north-west source MM3 is particularly strong, and the polarised signal at its peak is $10 \%$ of the total intensity. This value must be considered as an upper limit because of the flux loss, which is higher in the stokes $I$ than in the polarised signal. A zoomin of the four cores is given in Figs. 4 and 5, where the black contours represent the linear polarised emission and the white segments denote the magnetic field, where we assumed that the magnetic field is perpendicular to the linear polarisation vectors. The sampling of the vectors, only in these figures, are every 165 by 165 mas. At our densities and at the given scales the polarised signal is dominated by magnetic field effects and not by scattering (Kataoka et al. 2016, 2017). The polarisation vectors in each of the six cores exhibit an ordered structure indicating that 

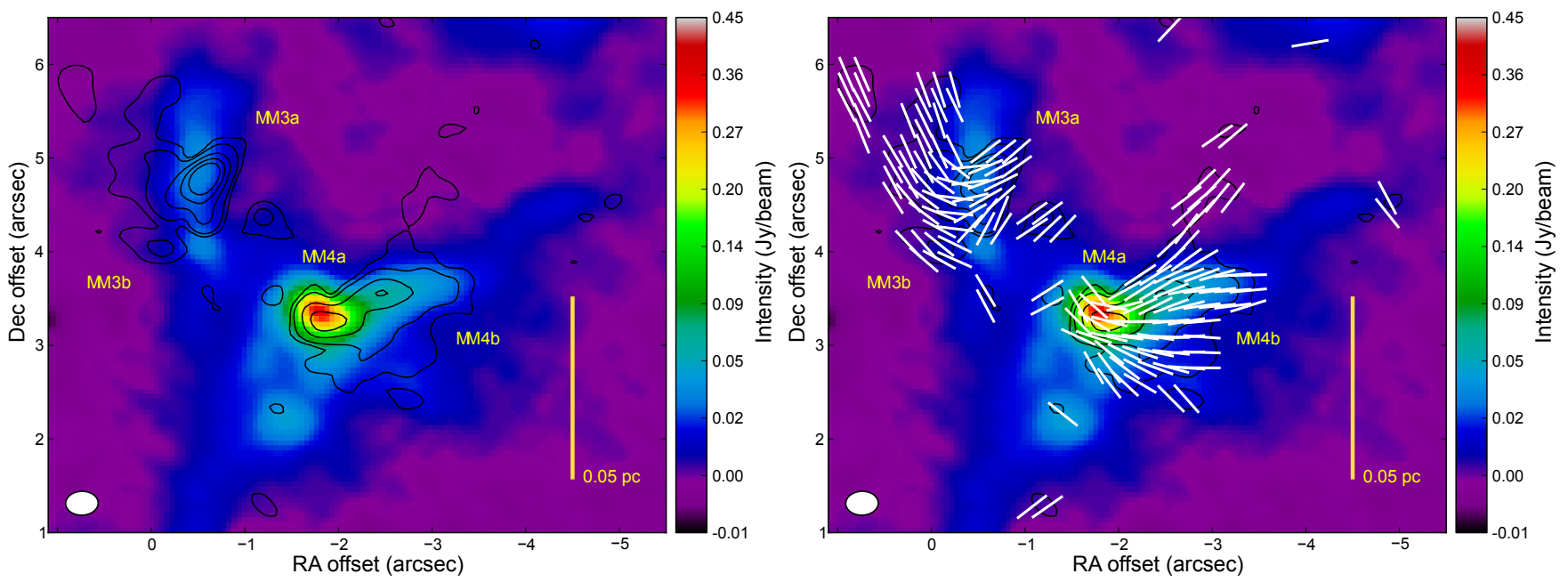

Fig. 4. Zoom on the cores MM3 and MM4 with the relative subcores. Left panel: the contours represent linearly polarised emission and the levels are $(0.3,0.5,1.0,1.5,2.0) \mathrm{mJy} \mathrm{beam}^{-1}$. Right panel: the segments indicate linear polarisation vectors already rotated of $90^{\circ}$ to show the orientation of the magnetic field. Only in this figure, the sampling of the vectors are every 165 by 165 mas. In both, the offsets are relative to the same position as in Fig. 3 and the colour scale indicates the total intensity of the background image in Jy beam ${ }^{-1}$, going from -0.01 to $0.45 \mathrm{Jy}$ beam ${ }^{-1}$; the yellow bar indicates the physical scale of $0.05 \mathrm{pc}$, at the distance of the source.

the magnetic field may be important in these sources, because, in case of turbulence dominated regions, the polarisation vectors distribution would have been less ordered. While in all the cores in MM3 and MM4, and in MM7, MM8c, MM9, and MM11a the polarisation vectors show smooth angle shifts, in the more extended region including the subcores MM8a and MM8b the polarisation angle distribution resembles a spiral structure. Nevertheless, the magnetic field morphology of the whole region seems to follow the direction of the stream of dust along the filament. In Fig. 5 the dotted yellow circle indicates the inner one-third of the primary beam (as already described in Fig 2). Following ALMA recommendations we have excluded from our analysis the polarised emission coming from MM6 and MM11c, since they are located beyond the one-third of the primary beam.

\subsection{Magnetic field strength}

\subsubsection{Davis-Chandrasekhar-Fermi method}

The magnetic field strength in a gas can be inferred by applying the Davis-Chandrasekhar-Fermi (DCF) method (Davis \& Greenstein 1951; Chandrasekhar \& Fermi 1953), following the procedure described in Planck Collaboration Int. XXXV (2016). Given the volume gas density $\rho$, the angular dispersion of the local magnetic field orientation $\sigma_{\psi}$ and the one-dimensional velocity dispersion of the gas $\sigma_{\nu}$, the strength of the magnetic field component in the plane of the sky in Gauss is

$B_{\perp}^{\mathrm{DCF}}=\xi \sqrt{4 \pi \rho} \frac{\sigma_{v}}{\sigma_{\psi}}$

where we assume that the magnetic field is frozen in the medium and the dispersion of $\sigma_{\psi}$ is due to transverse and incompressible Alfvén waves. In Eq. (9), $\rho=\mu n m_{H}$ is in $\mathrm{g} \mathrm{cm}^{-3}, \sigma_{v}$ is $\mathrm{cm} \mathrm{s}^{-1}$ and $\sigma_{\psi}$ is in rad. The correction factor $\xi$ is usually taken as $0.3 \leq \xi \leq 0.5$ and it has been derived by simulations of MHD turbulence in molecular clouds (Ostriker et al. 2001; Padoan et al. 2001; Heitsch et al. 2001; Falceta-Gonçalves et al. 2008). To avoid an overestimate of $B_{\perp}^{\mathrm{DCF}}, \xi$ must be applied in case of strong magnetic field (i.e. $\sigma_{\psi} \leq 25^{\circ}$ ). Crutcher et al. (2003) however pointed out that self-gravitating cores were not properly resolved in those simulations, since the simulations were halted after the formation of dense filaments because of insufficient resolution to follow the evolution further (Crutcher et al. 2003). Moreover, Cho \& Yoo (2016) argued that in the presence of averaging (e.g. on $\sigma_{\psi}$ along the line of sight or on the polarisation angle within the telescope beam) the DCF method tends to overestimate the magnetic field strength and thus they proposed a correction factor $0.7 \leq \xi \leq 1.0$. Therefore, considering all these effects, we decided to use a correction factor $\xi=0.5$, as proposed by Crutcher et al. (2003). We also assume a mean molecular weight of $2.8 m_{\mathrm{H}}$ and we use an average number density $n=$ $10^{6} \mathrm{~cm}^{-3}$ for denser cores MM4 and MM8, while $n=10^{5} \mathrm{~cm}^{-3}$ for less dense cores MM3 ( $n$ are from L17).

The angular dispersion of the local magnetic field orientations has been estimated over the selected pixels in each POLI images, considering

$\begin{aligned} \sigma_{\psi} & =\sqrt{\left\langle(\Delta \psi)^{2}\right\rangle}, \\ \Delta \psi & =0.5 \times \arctan \left(\frac{Q\langle U\rangle-\langle Q\rangle U}{Q\langle Q\rangle-\langle U\rangle U}\right),\end{aligned}$

where $\psi$ is the polarisation orientation angle.

The L17 authors observed some molecular species emitted from the same cores that we observed in our data. We make use of $\mathrm{CH}_{3} \mathrm{OH}$ lines from $\mathrm{L} 17$ (either from their Table 2 or their spectra) to estimate the velocity dispersion $\sigma_{v} \sim \Delta v / \sqrt{8 \ln 2}$. Then we calculate the $B_{\perp}^{\mathrm{DCF}}$ for the cores MM3, MM4, MM8, whose values are reported in Table 2.

The formal uncertainties on the magnetic field strength are computed considering the errors propagation on the angle and velocity dispersion. However the absolute uncertainties for the DCF method are larger because of the uncertainties on the density $n$, which we assume to be in the range $n=10^{5} \sim 10^{6} \mathrm{~cm}^{-3}$, considering the error on the correction factor of the order of $30 \%$ (Crutcher et al. 2003).

The estimates of $\sigma_{\psi}$ were performed using the "classical" variance, calculated around the circular mean (Jammalamadaka \& SenGupta 2001, Pewsey et al. 2013), selecting an individual region for MM3, MM4, and MM8. MM4 presents a central core and it is elongated to the west. The L17 work does not report 

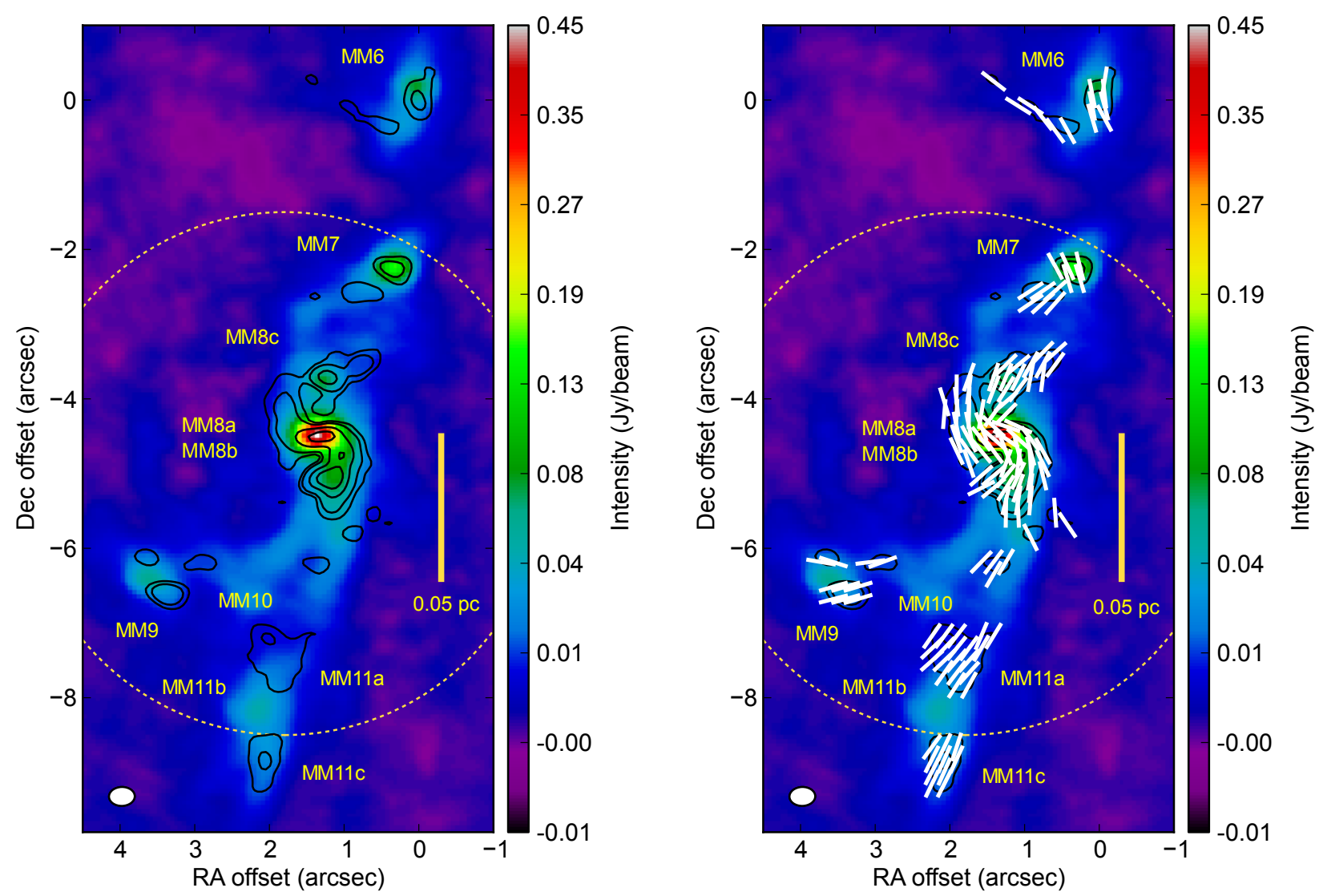

Fig. 5. Zoom on the core MM8 and relative subcores. Left panel: the contours represent linearly polarised emission and the levels are (0.3, 0.5, 1.0, $1.5,2.0) \mathrm{mJy}_{\text {beam }}{ }^{-1}$. Right panel: the segments indicate linear polarisation vectors already rotated of $90^{\circ}$ to show the orientation of the magnetic field. Only in this figure, the sampling of the vectors are every 165 by 165 mas. In both, the offsets are relative to the same position as in Fig. 3 and the colour scale is relative to the background image that indicates the total intensity in Jy beam ${ }^{-1}$, going from -0.01 to $0.45 \mathrm{Jy}_{\text {beam }}^{-1}$; the yellow bar indicates the physical scale of $0.05 \mathrm{pc}$, at the distance of the source. The dotted yellow circle indicates the inner one-third of the primary beam (as already described in Fig. 2).

any velocity dispersion for MM3, therefore we assumed the same values of velocity dispersion used for MM4. MM8 presents also a complex structure that has two filaments of gas with the shape of spiral arms around a central core. MM7, MM8c, MM9, and MM11a are very compact sources, showing no specific structure. They are too close to a point source to obtain reasonable estimates of the magnetic field using them alone. Therefore, we estimated $\sigma_{\psi}$ considering only the entire core MM8.

However, the magnetic field values obtained from this method should be considered as order-of-magnitude estimates because they do not fully encompass the complexity of the field dynamics in each part of the protostar. For example, the value of $\sigma_{\psi}$ is an average of magnetic field vectors and it is really difficult to determine the region in which this average must be computed, especially in sources showing complicated structures such as G9.62. In addition it is not clear which turbulent velocity is relevant for the dust (we used $\mathrm{CH}_{3} \mathrm{OH}$ in Table 2) and this results in one more uncertainty in the DCF method.

Following Planck Collaboration Int. XXXV (2016), in Fig. 6 we plot the histogram of the distribution of polarisation orientation angles $\psi$ towards the regions of MM3, MM4, and MM8. Each bin is $10^{\circ}$ wide. From the plot, all the cores present a large distribution of polarisation orientation angles. A broad distribution of polarisation orientation angles can imply an overestimate of $\sigma_{\psi}$ and consequently it can cause an underestimate of the magnetic field. For example, MM8 exhibits a spiral-shaped pattern of the polarisation segments resulting in a histogram with a large distribution of $\psi$ and its magnetic field is probably underestimated.

Moreover, the magnetic field structure inside a dense molecular cloud may be subject to effects that are not considered in the DCF analysis, such as differential rotation, gravitational collapse, or expanding HII regions. Therefore, we may observe a distortion in polarisation position angles due to large-scale non-turbulent effects. As a consequence, the dispersion values measured about mean fields, assumed to be straight, can be much larger than should be attributed to MHD waves or turbulence (Hildebrand et al. 2009; Houde et al. 2009).

\subsubsection{Structure function of the polarisation orientation angles}

A possible way to overcome the problem of the underestimate of $B_{\perp}^{\mathrm{DCF}}$ is by measuring the structure function (SF), also known as dispersion function of the polarisation orientation angles (Hildebrand et al. 2009; Houde et al. 2009; Koch et al. 2010). It consists in computing the mean absolute difference between polarisation angles as a function of their displacement $l$. Large values of the SF indicate large variations, while small values express a small dispersion between measured polarisation angles. The advantages of this method are that the SF does not depend on any model of the large-scale field. Nevertheless, it can only be applied under the same conditions as the DCF method, i.e. a smooth low-noise polarisation image, well-known 

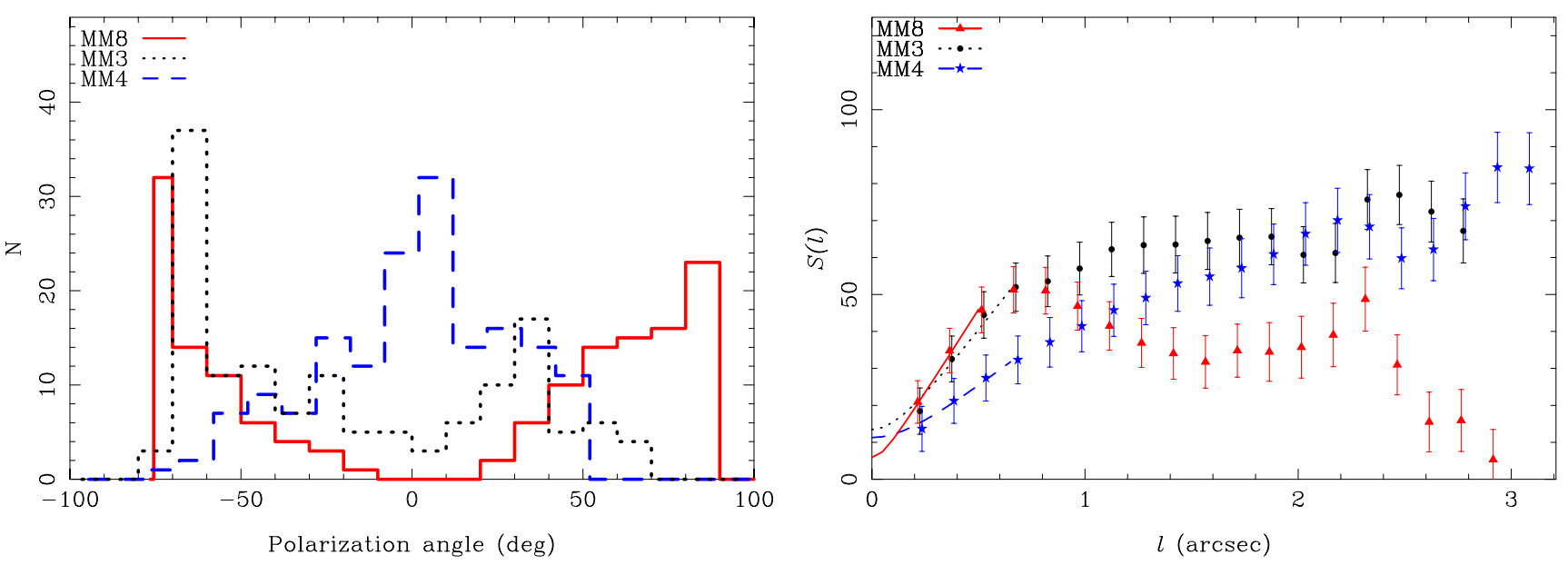

Fig. 6. Left panel: histograms of polarisation orientation angle $\psi$, towards the sources MM3, MM4, and MM8. Nyquist sampling was applied and each bin is $10^{\circ}$ wide. Right panel: square root of the SF of the polarisation orientation angles, $S(l)$ for the cores MM3, MM4, and MM8 represented by the black dots, blue stars, and red triangles, respectively. The best fits for MM3, MM4, and MM8 are plotted as the dotted black line, dashed blue line, and solid red line, respectively. In the SF plot, we applied Nyquist sampling and a bin of $0.15^{\prime \prime}$.

densities, and moderately uniform gas velocities. This method, however, provides an estimate of the turbulence to large-scale magnetic field strength ratio. Following Hildebrand et al. (2009), Houde et al. (2009), and Koch et al. (2010), in the right panel of Fig. 6 we plot the magnetic field dispersion $S(l)$, which is the square root of the SF of the polarisation orientation angles towards each source $S^{2}(l)=b^{2}+m^{2} l^{2}+\sigma_{\mathrm{M}}^{2}(l)$, where $b$ represents the turbulent contribution to the angular dispersion, $m^{2}$ is a constant, and $\sigma_{\mathrm{M}}^{2}(l)$ is the uncertainty on the polarisation angles. We consider a displacement $l>0.15^{\prime \prime}$ to avoid length scales smaller than the beam. In the plot, the data are binned with a width of $0.15^{\prime \prime}$ and using Nyquist sampling. The errors are computed as the standard deviation of the values inside the bin. The magnetic field is composed by a large-structured field $B_{0}$ and by a random component $B_{\mathrm{t}}$ and their relationship is given by

$\frac{B_{\mathrm{t}}}{B_{0}}=\frac{b}{\sqrt{\left(2-b^{2}\right)}}$.

Considering the same assumptions valid for the DCF method, i.e. incompressible and isotropic turbulence, magnetic field frozen into the gas, and dispersion of the $B_{\perp}$ orientation originating in transverse incompressible Alfvén waves,

$\frac{B_{\mathrm{t}}}{B_{0}}=\frac{\sigma_{v}}{\sigma_{\mathrm{A}}}$,

where $\sigma_{\mathrm{A}}=B_{0}(4 \pi \rho)^{-\frac{1}{2}}$ is the Alfvén velocity.

Combining Eqs. (12) and (13) we obtain the magnetic field component on the plane of the sky computed using the SF method

$B_{\perp}^{\mathrm{SF}}=\sqrt{4 \pi \rho} \frac{\sigma_{v} \sqrt{2-b^{2}}}{b}$.

When $B_{\mathrm{t}} \ll B_{0}$, then Eq. (14) can be approximated

$B_{\perp}^{\mathrm{SF}} \simeq \sqrt{8 \pi \rho} \frac{\sigma_{v}}{b}$

For the details of the mathematical demonstration of the previous formulae, we refer to Appendix A in Hildebrand et al. (2009) and Appendix D in Planck Collaboration Int. XXXV (2016). By fitting $S^{2}(l)$, we can derive the intercept of the fit $b^{2}$, and its square root gives an alternative measure for the dispersion of polarisation angles $\sigma_{\psi}$ (see Table 2). In Table 2 we also report the magnetic field component on the plane of the sky computed using the SF method and the ratio $B_{\mathrm{t}} / B_{0}$ showing that the large-scale magnetic field dominates the turbulent component. We considered the regions MM3, MM4, and MM8. As described in Houde et al. (2009) and Koch et al. (2010), we estimated the number of turbulent cells $N \sim 2$ from

$N=\frac{\left(\delta^{2}+2 W^{2}\right) \Delta^{\prime}}{\sqrt{2 \pi} \delta^{3}}$,

where $W$ is beam radius, $\Delta^{\prime}$ is the effective depth of the molecular cloud along the line of sight, and $\delta$ is the turbulent correlation length. Considering the distance of our source, our beam radius $\left(\sim 0.15^{\prime \prime}\right)$ results in a physical size of $W \sim 3.7 \mathrm{mpc}$. We assumed a $\Delta^{\prime} \sim 40 \mathrm{mpc}$, which is approximately the size of our cores and a $\delta \sim 10 \mathrm{mpc}$, which is comparable to estimates made for other similar cores (Girart et al. 2013; Frau et al. 2014; Juárez et al. 2017). Therefore, the beam effect correction results $\sim \sqrt{N}=1.4$, which does not produce a significant change in our $B_{\mathrm{t}} / B_{0}$ ratio. This is consistent with the view proposed by Koch et al. (2010), where the beam correction is important for low-resolution data, but it is less important for high-resolution observations.

\subsubsection{Turbulent-to-magnetic field energy ratio}

We can determine the one-dimensional Alfvén velocity $\sigma_{\mathrm{A}} \sim 15 \mathrm{~km} \mathrm{~s}^{-1}$, assuming that the component along the line of sight is the same on the plane of the sky and considering an approximate mean magnetic field strength in the plane of the sky of the order of $11 \mathrm{mG}$ (assumed from $\mathrm{B}_{\perp}^{\mathrm{SF}}$ values in Table 2 ) and a typical number density of $10^{6} \mathrm{~cm}^{-3}$ (L17).

The comparison of $\sigma_{\mathrm{A}}$ with the one-dimensional velocity dispersion for each individual core $\sigma_{v}$ (Table 2) indicates that any turbulent or infall velocity is below the Alfvénic velocity. The ratio $\sigma_{v} / \sigma_{\mathrm{A}} \sim 0.16$ is consistent with the values that we find for $B_{\mathrm{t}} / B_{0}$ ratio. We estimate the ratio of turbulent-to-magnetic energy as $\gamma \sim 3\left(\sigma_{v} / \sigma_{\mathrm{A}}\right)^{2}$ (Beuther et al. 2018; Girart et al. 2009). Using the larger line-of-sight velocity dispersion found for the cores in MM8 $\left(\sigma_{v}=2.35 \mathrm{~km} \mathrm{~s}^{-1}\right)$, the turbulent-to-magnetic energy ratio is $\gamma \sim 0.07$. Thus, the magnetic energy along the 
filament appears to dominate over the turbulent energy indicating that magnetic field seems to be playing a more dominant role than turbulence.

The important role played by the magnetic field might also influence the morphology and fragmentation of the filament, resulting in a tiny number of low-mass protostars. Simulations performed by Inoue \& Fukui (2013) suggested that because of enhanced magnetosonic speed and turbulence behind the shock, the effective Jeans mass and the mass accretion rate can have a larger value in this region, triggering the formation of mainly massive cores. From their simulations, which include MHD contributions, massive star formation is naturally triggered when the massive filament collapses globally. However, the lack of low-mass protostars along the filament could also be justified considering other theories such as the "collect and collapse" process, as discussed in L17. External heating coming from the two $\mathrm{H}$ II regions (B and $\mathrm{C}$ see Fig. 1) and internal heating generated by outflows from hot molecular cores (such as MM8 or MM4) increased the thermal Jeans masses and consequently suppressed the fragmentation.

\subsection{Mass to magnetic flux ratio}

Another way to quantify if the magnetic field can affect the fragmentation and formation of new stars is to estimate the mass-toflux ratio $\left(M / \Phi_{\mathrm{B}}\right)$. This ratio defines the stability of a region and whether a static magnetic field can support a cloud against gravitational collapse (Crutcher 1999; Troland \& Crutcher 2008). The mass-to-flux ratio in units of critical mass-to-flux ratio is

$\lambda=\left(M / \Phi_{\mathrm{B}}\right)_{\mathrm{obs}} /\left(M / \Phi_{\mathrm{B}}\right)_{\text {crit }} \sim 7.6 \times 10^{-24} N_{\mathrm{H}_{2}} / B$,

where $\left(M / \Phi_{\mathrm{B}}\right)_{\text {crit }}$ and $\left(M / \Phi_{\mathrm{B}}\right)_{\text {obs }}$ are the theoretically determined critical mass-to-flux ratio (Nakano \& Nakamura 1978) and the observed mass-to-flux ratio, respectively, with $N_{\mathrm{H}_{2}}$ in $\mathrm{cm}^{-2}$ and $B$ in $\mathrm{mG}$.

Using the column densities and the average magnetic field obtained from the SF analysis reported in Tables 1 and 2, respectively, we get $\lambda$ varying roughly from 8 to 13 . Looking at the mass-to-magnetic-flux ratio, the G9.62 clump appears to be super-critical, which is consistent with Cortes \& Crutcher (2006) because $\lambda$ is several order of magnitude above the critical value and indeed star formation is occurring in the filament.

However, the column density used to determine $\lambda$ naturally implies an average density that is much higher than the density adopted in the magnetic field calculation (Eqs. (9) and (15)), when assuming a source size along the line of sight similar to that on the plane of the sky. Hence, the magnetic field strength measured is not necessarily representative of the magnetic field supporting the core, which means that $\lambda$ is strictly an upper limit and could be more than an order of magnitude smaller. This illustrates one of the main uncertainties of estimating $\lambda$ from dust polarisation measurements. In addition, Eq. (17) comes from cloud models computed from initially uniform, spherical clouds with initially uniform magnetic fields. If the mass is differently funnelled along magnetic flux lines, then it results in a different $\left(M / \Phi_{\mathrm{B}}\right)_{\text {crit }}$, which can be higher by a factor of 2 (Mouschovias 1991). Moreover, if the cores are supported primarily by static magnetic fields, they should have flattened structures and therefore $N_{\mathrm{H}_{2}}$ should be measured parallel to the magnetic field to correctly estimate the mass-to-flux ratio.

Thus, taking into account all the approximations and uncertainties on $\lambda$, on the column densities and on the magnetic field strength obtained with the SF method, these values of the mass-to-magnetic-flux ratio should be considered with caution.

\subsection{Detection of thermal line polarisation}

In one of the spectral windows, molecular emission was detected from the core MM4a with a peak at $337.6 \mathrm{GHz}$. We performed the continuum subtraction and we detected linearly polarised emission only from the core MM4a and in few channels around the peak of the line emission. Therefore we exclude any dependence on the position within one-third of the primary beam. In our observations we do not have enough spectral resolution to identify unambiguously the molecular species. Considering for MM4a a velocity of $\sim 2 \mathrm{~km} \mathrm{~s}^{-1}$ (from L17), methanol or sulphur dioxide are the most likely candidates, since they are species that have already been observed in the core (L17) and both of these present several lines at the frequency of the observed peak.

In Fig. 7 we plot the total line intensity, linear polarisation contours, and vectors indicating the polarisation direction already rotated of $90^{\circ}$. It is ambiguous, for the polarisation resulting from the Goldreich-Kylafis effect, whether the magnetic field orientation is identical to the polarisation orientation or whether a $90^{\circ}$ rotation is necessary. We decided to plot the polarisation vectors already rotated by $90^{\circ}$. This decision was made under a few assumptions based on the analysis performed by Cortes et al. (2005) for the $\mathrm{CO}$ line. The linear polarisation can be perpendicular to the magnetic field lines in presence of a velocity gradient which is smaller in the direction parallel to the magnetic field. If we consider the direction of the magnetic field probed by our dust observations and we look at the direction of the velocity gradient in Fig.5 in L17, we see that this condition is valid for MM4. We are then assuming an anisotropy of the radiation field which can allow an emission polarised in the direction perpendicular to the magnetic field. However, we emphasise that we are conscious of the ambiguous behaviour of the polarisation resulting from the Goldreich-Kylafis effect and that the work by Cortes et al. (2005) was proposed for another molecule. Even if we do not have firm information about the radiation field, we present the direction already rotated by $90^{\circ}$ to show a tentative comparison between the three independent methods to infer the magnetic field (see Sect. 5.2). For a more complete analysis, more detailed knowledge about the radiation field and the optical depth of the molecule is needed.

The total intensity peak is $0.52 \mathrm{Jy} \mathrm{beam}^{-1}$, and the linear polarised intensity is $2.38 \mathrm{mJy}^{\text {beam }}{ }^{-1}$. The polarisation threshold is $1.4 \mathrm{mJy} \mathrm{beam}^{-1}$ and the rms is $0.4 \mathrm{mJy} \mathrm{beam}^{-1}$. The direction of the linear polarisation is consistent with that of core MM4a observed with the dust, while it is perpendicular to the direction of the vectors marking the elongated structure in northeast direction. Because of the lack of spectral resolution and the uncertainty in the identification of the molecule, we do not further analyse the $336.7 \mathrm{GHz}$ line.

\section{Discussion}

\subsection{Magnetic fields in different evolutionary stages}

Since complex organic molecules are good tracers of core evolution (Qin et al. 2010), L17 suggested that the presence of molecules such as $\mathrm{CH}_{3} \mathrm{OH}$ and $\mathrm{CH}_{3} \mathrm{OCHO}$ in MM4 and MM8 (and relative subcores) indicates that both cores are in a hot 

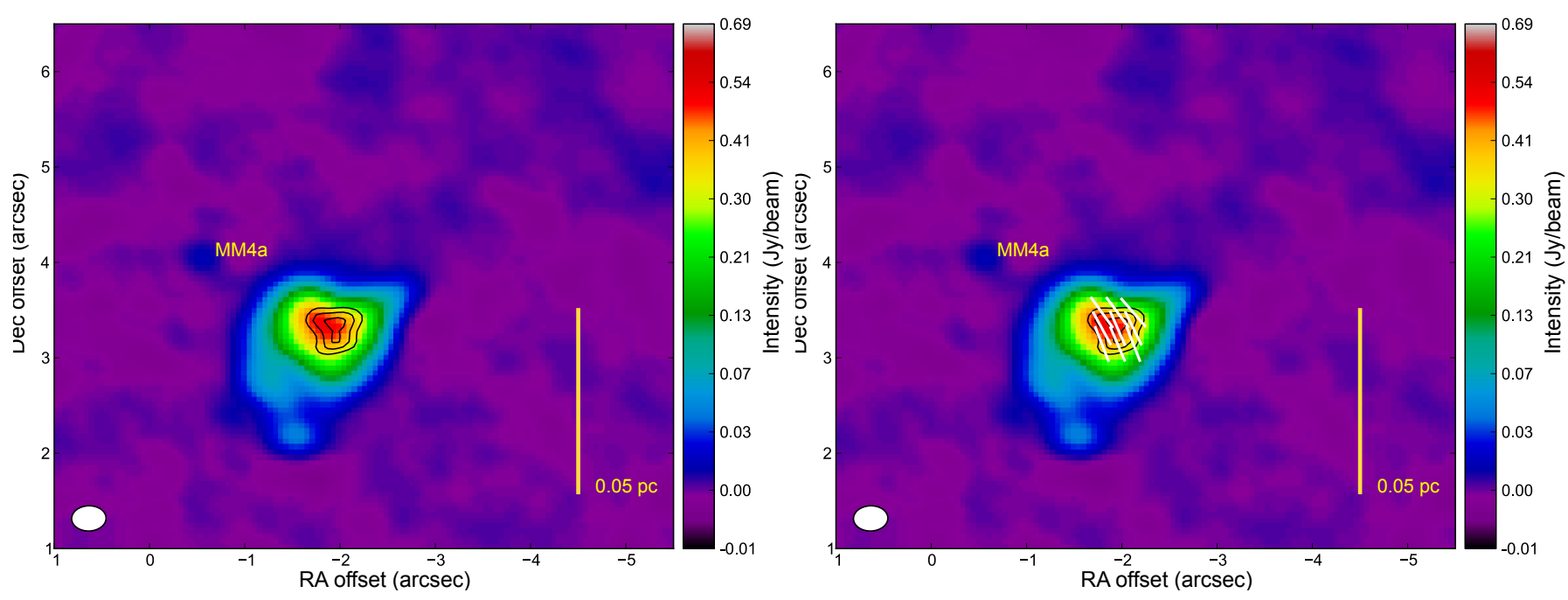

Fig. 7. Total intensity image of the thermal line emitted in the core MM4a. Left panel: the contours represent linearly polarised emission and the levels are $(1.4,1.9,2.3) \mathrm{mJy}_{\text {beam }}{ }^{-1}$. The linear polarisation peak intensity is $2.38 \mathrm{mJy}$ beam $^{-1}$. Right panel: the segments indicate linear polarisation vectors already rotated by $90^{\circ}$ to show the orientation of the magnetic field. The sampling of the vectors are every 165 by 165 mas. In both panels, the offsets are relative to the same position as in Fig. 3 and the colour scale indicates the total intensity in Jy beam ${ }^{-1}$, going from -0.01 to $0.52 \mathrm{Jy} \mathrm{beam}^{-1}$; the yellow bar indicates the physical scale of $0.05 \mathrm{pc}$ at the distance of the source.

molecular core phase ${ }^{2}$. Moreover, again according to L17, MM8 is less evolved than MM4 because MM8 is driving energetic outflows and it might be still in accretion phase. MM3 is instead a starless core, probably at an early evolutionary phase (L17).

Therefore the evolutionary sequence from less evolved core to more evolved core is MM3 $\rightarrow$ MM8 $\rightarrow$ MM4. Depending on the exact evolutionary stage, we could expect to observe highly ordered magnetic fields in the more quiescent cores, while the field in the more evolved protostars is less uniform. As shown in Figs. 4 and 5, our band 7 observations reveal an elongated structure for MM3, MM4, and MM8 and several subcores showing polarised emission.

In MM3 we detected the strongest linear polarisation fraction observed in G9.62 and the field lines are organised in a orderly pattern. However in MM3 the resulting $B_{\perp}$ is probably underestimated since, to perform the magnetic field analysis, we assumed the same emission of $\mathrm{CH}_{3} \mathrm{OH}$ observed in MM4 and L17 did not report any line for this core. Moreover, although MM3 shows a highly polarised flux, its spectral index is not really different from the other cores. Therefore, it may be possible that MM3 is at an early evolutionary phase and the collapse did not start yet because gravity was unable to overcome magnetic pressure in this core.

MM4 is the most evolved core (L17 and references therein), since it shows a strong continuum emission and no molecular outflow. It presents a elongated profile in the north-west direction and the linear polarisation vectors show a orderly patter. MM4 shows a broad distribution of $\psi$ (Fig. 6) but only one peak, indicating the presence of one component and a less complex magnetic field. From Fig. 5 in L17, we can also see that MM4 presents a range of velocities from $\sim 1$ to $\sim 3 \mathrm{~km} \mathrm{~s}^{-1}$.

2 Liu et al. (2017) performed SED fittings and obtained archived data from Herschel $(70,160,250,350$, and $500 \mu \mathrm{m})$, JCMT/SCUBA (450 and $850 \mu \mathrm{m}$ continuum), APEX/LABOCA (875 $\mu \mathrm{m}$ continuum images), and SEST/SIMBA (1.2 mm continuum image). For the spectral lines analysis they made use of ALMA band 6 data. All the details are reported in Appendix A in L17. For further references see also Sect. 2.
MM8 is in an early hot core stage showing outflows and clear signatures of accretion (L17). In our total intensity image, MM8 exhibits a structure along the north-south direction and the polarisation vectors are organised in a peculiar spiral shape. This could be a signature of compressed magnetic field due to infalling material that is accreted by the young protostars, and the magnetic field lines are still frozen in the medium. The broad distribution of polarisation orientation angles that we find in Fig. 6 could be due to the presence of separate components. From Fig. 5 in L17, we can also see that MM8 presents a range of velocities broader than MM4, spanning from $\sim 1$ to $\sim 6 \mathrm{~km} \mathrm{~s}^{-1}$.

Since we do not have molecular lines observations at the same resolution in the same region in our Band 7 data, we could not perform any velocity field analysis. We decided to exclude MM3 from the comparison, since its magnetic field could be underestimated. Thus, we focussed only on MM8 and MM4; interpreting the magnetic field data in terms of the evolutionary stages, we observe a magnetic field stronger in the youngest core MM8 than in the more evolved source MM4. This is consistent with the scenario in which magnetic fields are influencing star formation and it is symptomatic of an evolution of the magnetic field strength and morphology during the entire star formation process.

At small scales of $0.1 \mathrm{pc}$, all the cores in MM3, MM4, and MM8 present magnetic fields that are organised and non-chaotic. Comparing the direction of the outflows studied by L17 with the direction of the magnetic field indicated by our linear polarisation observations, we see that they are perpendicular, which is partially in disagreement with the finding of Hull et al. (2013). Since there is a correlation between the orientation of the axes of the molecular outflow and the magnetic field, it could be a signature of a magnetically regulated evolution. The magnetic field could be strong enough to funnel the gas along the field lines and regulate the accretion and disc orientation.

Furthermore, it seems that the cores presenting a uniform magnetic field, such as MM3 or MM4, are less fragmented than the other cores not showing polarisation. We also estimate on average a turbulent-to-magnetic energy ratio of $\gamma \sim 0.07$, indicating that the magnetic energy along the filament dominates over 
the turbulent energy. Moreover the orientations of the magnetic field segments in our ALMA observations (core-scale) are consistent with those in JCMT/POL-2 observations (clump-scale) (Liu et al. 2018). Therefore, this can be a further evidence that magnetic fields have a strong influence on the star formation processes in this clump, playing an important role in the collapse and fragmentation phase.

\subsection{Tentative comparison between dust continuum, thermal line, and maser data}

In this section we want to discuss a tentative comparison of the resulting magnetic field for the G9.62 clump obtained by three different and independent methods: the analysis of the linear polarised light emitted by dust in the continuum (DCF and SF methods), the analysis of the linear polarised light emitted by thermal molecular transitions, and the analysis of the polarised maser emission.

In Fig. 8, we plot a summary of the magnetic field directions obtained from our ALMA $348 \mathrm{GHz}$ data and from $1.6 \mathrm{GHz} \mathrm{OH}$ masers observations (Fish et al. 2005a; Fish \& Reid 2006). In this figure, the linear polarisation segments are overplotted on the total intensity image of the filament. The segments are already rotated by $90^{\circ}$, indicating the direction of the magnetic field as inferred by different observations at different wavelengths. In general, the magnetic field exhibits an ordered structure along the direction of this filament. Comparing the direction of the outflows studied by L17 with the direction of the magnetic field denoted by our linear polarisation observations, we see that they are perpendicular. In addition, if we assume a rotation of $90^{\circ}$, the linear polarisation vectors obtained from the thermal line analysis in the core MM4a point the same direction indicated by the polarised dust (Fig. 8). Moreover, the averaged magnetic field strength on the plane of the sky obtained from our analysis (of the order of $11 \mathrm{mG}$ ) is comparable with the component along the line of sight, already proposed by $\mathrm{OH}$ and $\mathrm{CH}_{3} \mathrm{OH}$ masers observations (Vlemmings 2008). Indeed $\mathrm{OH}$ maser Zeeman splitting observations indicate a magnetic field strength of $\sim 5 \mathrm{mG}$. The magnetic field determined from the $6.7 \mathrm{GHz}$ methanol masers also revealed a similar strength. The linear polarisation vectors from the $\mathrm{OH}$ masers appear consistent with our linearly polarised data, but unfortunately the small number of maser observations hinders a quantitative demonstration. Even though the thermal line, masers, and dust polarisation probe very different physical processes and likely very different volumes as well, observations towards the massive protostar IRAS18089-1732 (Dall'Olio et al. 2017; Beuther et al. 2010) showed that the same magnetic field can affect both the small scale of few astronomical units around the protostar (probed by masers) and the large scale of the torus (probed by dust). The alignment of the geometry between the dust emission (coming from the core) and the thermal line emission (coming from the envelope) has also been found in DR21(OH) (Lai et al. 2003; Cortes et al. 2005), suggesting a connection between the magnetic field in the core and that in the envelope. So further observations of polarised thermal line, masers, and dust are needed to investigate the alignment of the magnetic field at different scales of star forming regions.

\section{Conclusions}

In this paper, we presented our investigation of the magnetic field morphology of the well-known high-mass star forming region G9.62+0.19. We analysed ALMA band 7 continuum observations and we identified 23 cores and substructures. For these we reported the position, peak flux densities, integrated flux, deconvolved major and minor axes, position angles, and spectral index.

We detected linear polarised emission from two cores and relative subcores in the northern field (MM3 and MM4) and from four in the southern field (MM7, MM8, MM9, MM11a). One of these, MM3a showed a linear polarisation fraction of $\sim 10 \%$. For these we studied the magnetic field strength on the plane of the sky component $B_{\perp}$, comparing the DavisChandrasekhar-Fermi (DCF) and the structure function (SF) analysis. Through the SF we derived estimates of the magnetic field strength along the line of sight that were larger than the values obtained by the classical DCF method.

By comparison with band 6 observations (Liu et al. 2017), for some of the cores we obtained the spectral index $\alpha$ and the spectral index for the dust opacity $\beta$ and we estimated the $N_{\mathrm{H}_{2}}$ column densities and the masses. We found that the core masses range roughly between $10 M_{\odot}$ and $40 M_{\odot}$ and the $N_{\mathrm{H}_{2}}$ column densities between $3 \times 10^{24}$ and $5 \times 10^{25} \mathrm{~cm}^{2}$. Because of the large uncertainties, mainly determined by the errors on the dust model, on the spectral index and optical depth, the errors of the masses and column densities are difficult to quantify and they could be more than a factor of 5 .

We proposed an evolutionary sequence for the magnetic field of the two hot cores MM4 and MM8, indicating that MM4 is more evolved than MM8 because of the presence of a weaker magnetic field than that observed in MM8. MM3 is a starless core at an early evolutionary phase and the collapse did not start yet, because in this core gravity was probably unable to overcome magnetic pressure yet; its magnetic field is indeed probably underestimated since from previous observations we do not have information about molecular lines coming from this core.

In general the magnetic field seemed to follow the direction of the filament, and it was perpendicular to the direction of the outflows emitted by some massive protostellar cores such as MM8a, MM7, and MM6. The cores that presented polarisation appeared to be less fragmented than those not showing polarised emission. At scales less than $0.1 \mathrm{pc}$, the magnetic field showed a neat and ordered pattern of polarisation vectors.

We also detected linearly polarised molecular line, thermally emitted probably by methanol or carbon dioxide. Moreover, from the SF analysis we obtained an average magnetic field strength of the order of $11 \mathrm{mG}$. The magnetic field strength on the plane of the sky obtained from our analysis is comparable with the component along the line of sight, already detected from previous $\mathrm{OH}$ and $\mathrm{CH}_{3} \mathrm{OH}$ masers observations on the core MM4b and MM7 (Fish et al. 2005a; Vlemmings 2008), suggesting that at scale of less of $0.05 \mathrm{pc}$ the magnetic field role could be important in this region.

We estimated the ratio of turbulent-to-magnetic energy and we found on average a turbulent-to-magnetic energy ratio of $\gamma \sim 0.07$, indicating that the magnetic energy along the filament dominates over the turbulent energy.

However, because of the uncertainties of the DCF and the SF methods, which are inevitably reflected on the column densities and masses, further investigations are needed to properly evaluate the magnetic field strength in the G9.62 region. The use of combined observations of masers, dust, and molecular lines contribute towards understanding the role of the magnetic field at different scales. As demonstrated by our observations, ALMA has the capabilities to detect the weak Goldreich-Kylafis effect (Goldreich \& Kylafis 1982) occurring in molecular lines. Therefore this instrument can add more details and constraints on the 


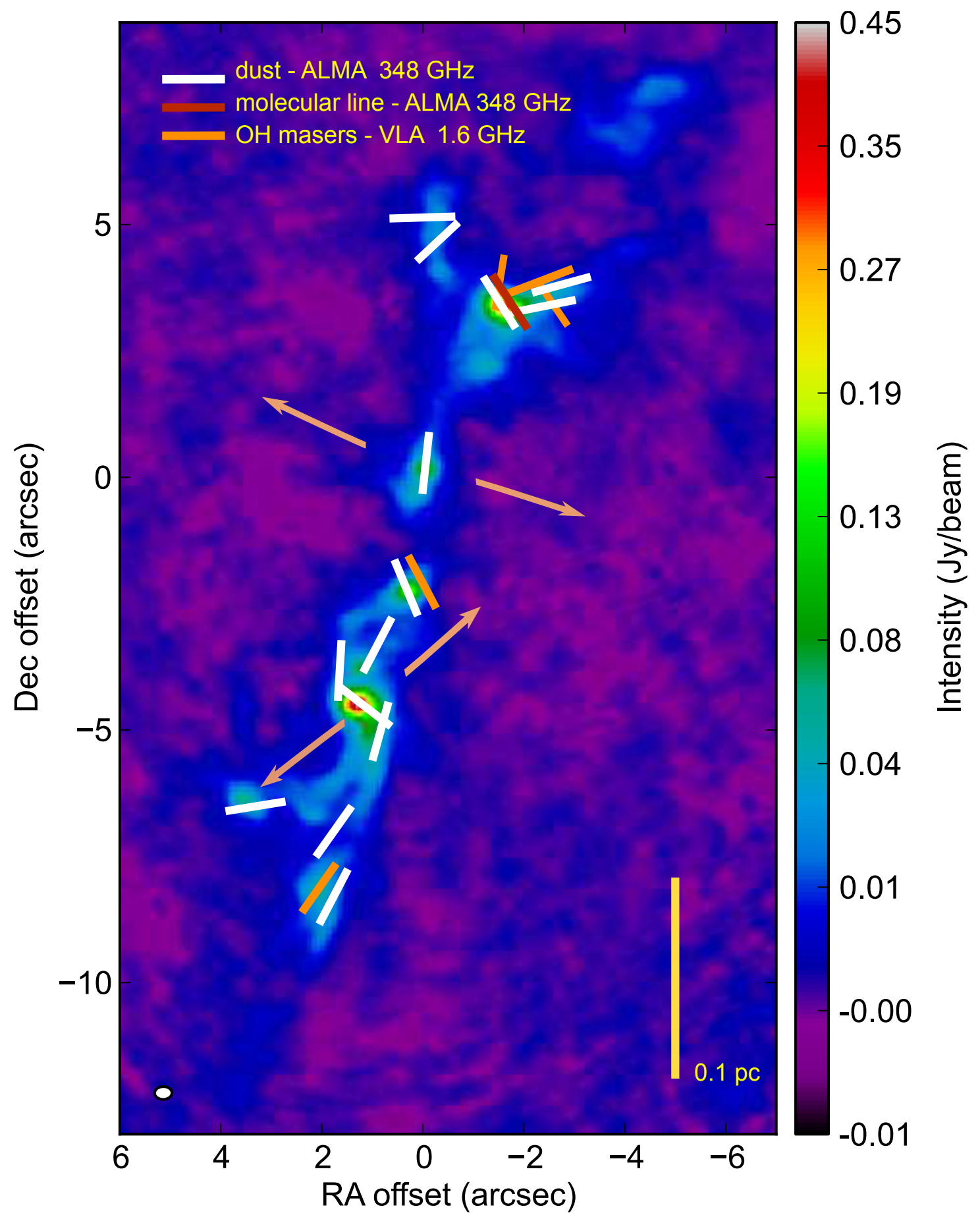

Fig. 8. Total intensity image of the star forming region G9.62+0.19 and linear polarisation segments, rotated by $90^{\circ}$, indicating the average direction of the magnetic field on scales of $\sim 0.5^{\prime \prime} \times 0.5^{\prime \prime}$. The different colours in the segments delineate the direction of the magnetic field as detected by different data at different wavelengths. The white and brown bars indicate dust observations and molecular thermal line, respectively, from our ALMA band 7 polarised data. The orange bars indicate OH maser linearly polarised observations (Fish et al. 2005a; Fish \& Reid 2006). Arrows denote the direction of the outflows (L17). The offsets are relative to the absolute position in Fig. 3. The white ellipse represents the beam. The colour scale goes from -0.01 to $0.45 \mathrm{Jy}_{\text {beam }}{ }^{-1}$ and the yellow bar indicates the physical scale of $0.1 \mathrm{pc}$, at the distance of the source.

magnetic field morphology and strength and can help to infer, for example, more precise mass-to-magnetic flux ratios.

Acknowledgements. We thank the referee Patrick Koch for the precise comments and suggestions that have contributed to the improvement of this work. The research leading to these results has received funding from the European Research Council under the European Union's Seventh Framework Programme (FP/2007-2013)/ERC Grant Agreement n. 614264. This paper makes use of the following ALMA data: ADS/JAO.ALMA \#2015.1.01349.S. ALMA is a partnership of ESO (representing its member states), NSF (USA) and NINS (Japan), together with NRC (Canada) and NSC and ASIAA (Taiwan) and KASI (Republic of Korea), in cooperation with the Republic of Chile. The Joint ALMA Observatory is operated by ESO, AUI/NRAO and NAOJ. D.D. thanks the Nordic ALMA Regional Centre (ARC) node based at Onsala Space Observatory for support, in particular Tobia Carozzi and Ivan Martí-Vidal. The Nordic ARC node is funded through Swedish Research Council grant No 2017-00648. D.D. also thanks Akimasa Kataoka and Kei Tanaka for interesting discussions. H.B. acknowledges support from the European Research Council 
under the Horizon 2020 Framework Program via the ERC Consolidator Grant CSF-648505. J.M.G. and J.M.T. are supported by the MINECO (Spain) AYA2017- 84390-C2 coordinated grant.

\section{References}

Alves, F. O., Girart, J. M., Padovani, M., et al. 2018, A\&A, 616, A56 Andersson, B.-G., Lazarian, A., \& Vaillancourt, J. E. 2015, ARA\&A, 53, 501 Attard, M., Houde, M., Novak, G., et al. 2009, ApJ, 702, 1584

Banerjee, R., \& Pudritz, R. E. 2007, ApJ, 660, 479

Beuther, H., Vlemmings, W. H. T., Rao, R., \& van der Tak, F. F. S. 2010, ApJ, 724, L113

Beuther, H., Soler, J. D., Vlemmings, W., et al. 2018, A\&A, 614, A64

Bonnell, I. A., \& Bate, M. R. 2006, MNRAS, 370, 488

Cesaroni, R., Churchwell, E., Hofner, P., Walmsley, C. M., \& Kurtz, S. 1994, A\&A, 288, 903

Chandrasekhar, S., \& Fermi, E. 1953, ApJ, 118, 113

Cho, J., \& Lazarian, A. 2005, ApJ, 631, 361

Cho, J., \& Yoo, H. 2016, ApJ, 821, 21

Commerçon, B., Hennebelle, P., \& Henning, T. 2011, ApJ, 742, L9

Cortes, P., \& Crutcher, R. M. 2006, ApJ, 639, 965

Cortes, P. C., Crutcher, R. M., \& Watson, W. D. 2005, ApJ, 628, 780

Crutcher, R. M. 1999, ApJ, 520, 706

Crutcher, R. M. 2012, ARA\&A, 50, 29

Crutcher, R. M., Nutter, D. J., \& Ward-Thompson, D. 2003, BASS, 35, 772

Cudlip, W., Furniss, I., King, K. J., \& Jennings, R. E. 1982, MNRAS, 200, 1169

Dall'Olio, D., Vlemmings, W. H. T., Surcis, G., et al. 2017, A\&A, 607, A111

Davis, Jr. L., \& Greenstein, J. L. 1951, ApJ, 114, 206

Draine, B. T., \& Weingartner, J. C. 1996, ApJ, 470, 551

Draine, B. T., \& Weingartner, J. C. 1997, ApJ, 480, 633

Falceta-Gonçalves, D., Lazarian, A., \& Kowal, G. 2008, ApJ, 679, 537

Fish, V. L., \& Reid, M. J. 2006, ApJS, 164, 99

Fish, V. L., Reid, M. J., Argon, A. L., \& Zheng, X.-W. 2005a, ApJS, 160, 220

Fish, V. L., Reid, M. J., \& Menten, K. M. 2005b, ApJ, 623, 269

Frau, P., Girart, J. M., Zhang, Q., \& Rao, R. 2014, A\&A, 567, A116

Garay, G., Rodriguez, L. F., Moran, J. M., \& Churchwell, E. 1993, ApJ, 418, 368

Girart, J. M., Rao, R., \& Marrone, D. P. 2006, Science, 313, 812

Girart, J. M., Beltrán, M. T., Zhang, Q., Rao, R., \& Estalella, R. 2009, Science, 324,1408

Girart, J. M., Frau, P., Zhang, Q., et al. 2013, ApJ, 772, 69

Girart, J. M., Fernández-López, M., Li, Z.-Y., et al. 2018, ApJ, 856, L27

Goldreich, P., \& Kylafis, N. D. 1982, ApJ, 253, 606

Heitsch, F., Zweibel, E. G., Mac Low, M.-M., Li, P., \& Norman, M. L. 2001, ApJ, 561, 800

Hildebrand, R. H. 1988, QJRAS, 29, 327

Hildebrand, R. H., Dragovan, M., \& Novak, G. 1984, ApJ, 284, L51

Hildebrand, R. H., Kirby, L., Dotson, J. L., Houde, M., \& Vaillancourt, J. E. 2009, ApJ, 696, 567

Hoang, T., \& Lazarian, A. 2008, MNRAS, 388, 117

Hofner, P., Wiesemeyer, H., \& Henning, T. 2001, ApJ, 549, 425

Houde, M., Vaillancourt, J. E., Hildebrand, R. H., Chitsazzadeh, S., \& Kirby, L. 2009, ApJ, 706, 1504

Hull, C. L. H., Plambeck, R. L., Bolatto, A. D., et al. 2013, ApJ, 768, 159

Inoue, T., \& Fukui, Y. 2013, ApJ, 774, L31

Inoue, T., Hennebelle, P., Fukui, Y., et al. 2018, PASJ, 70, S53

Jammalamadaka, S., \& SenGupta, A. 2001, Topics in Circular Statistics, Series on multivariate analysis (Singapore: World Scientific)

Johansen, A., \& Levin, Y. 2008, A\&A, 490, 501

Juárez, C., Girart, J. M., Zamora-Avilés, M., et al. 2017, ApJ, 844, 44

Kataoka, A., Tsukagoshi, T., Momose, M., et al. 2016, ApJ, 831, L12

Kataoka, A., Tsukagoshi, T., Pohl, A., et al. 2017, ApJ, 844, L5
Klassen, M., Pudritz, R. E., \& Kirk, H. 2017, MNRAS, 465, 2254

Klessen, R., Jappsen, K., Larson, R., Li, Y., \& Low, M.-M. M. 2005, Astrophys. Space Sci. Lib., 327, 363

Koch, P. M., Tang, Y.-W., \& Ho, P. T. P. 2010, ApJ, 721, 815

Lai, S.-P., Girart, J. M., \& Crutcher, R. M. 2003, ApJ, 598, 392

Lazarian, A. 2000, in Cosmic Evolution and Galaxy Formation: Structure, Interactions, and Feedback, eds. J. Franco, L. Terlevich, O. López-Cruz, \& I. Aretxaga, ASP Conf. Ser., 215, 69

Lazarian, A., \& Hoang, T. 2007, ApJ, 669, L77

Lazarian, A., \& Hoang, T. 2008, ApJ, 676, L25

Linz, H., Stecklum, B., Henning, T., Hofner, P., \& Brandl, B. 2005, A\&A, 429, 903

Liu, T., Wu, Y., Liu, S.-Y., et al. 2011, ApJ, 730, 102

Liu, T., Lacy, J., Li, P. S., et al. 2017, ApJ, 849, 25

Liu, T., Kim, K.-T., Liu, S.-Y., et al. 2018, ApJ, 869, L5

Machida, M. N., Inutsuka, S.-i., \& Matsumoto, T. 2014, MNRAS, 438, 2278

Matsushita, Y., Sakurai, Y., Hosokawa, T., \& Machida, M. N. 2018, MNRAS, 475,391

Maury, A. J., Girart, J. M., Zhang, Q., et al. 2018, MNRAS, 477, 2760

McKee, C. F., \& Tan, J. C. 2003, ApJ, 585, 850

McKee, C. F., \& Ostriker, E. C. 2007, ARA\&A, 45, 565

Mouschovias, T. C. 1991, Cosmic Magnetism and the Basic Physics of the Early Stages of Star Formation, eds. C. J. Lada, \& N. D. Kylafis (Dordrecht: Springer Netherlands), 61

Mouschovias, T. C., \& Paleologou, E. V. 1979, ApJ, 230, 204

Mouschovias, T. C., Tassis, K., \& Kunz, M. W. 2006, ApJ, 646, 1043

Myers, A. T., McKee, C. F., Cunningham, A. J., Klein, R. I., \& Krumholz, M. R. 2013, ApJ, 766, 97

Nakano, T., \& Nakamura, T. 1978, PASJ, 30, 671

Ossenkopf, V., \& Henning, T. 1994, A\&A, 291, 943

Ostriker, E. C., Stone, J. M., \& Gammie, C. F. 2001, ApJ, 546, 980

Padoan, P., \& Nordlund, ̊. 2002, ApJ, 576, 870

Padoan, P., Goodman, A., Draine, B. T., et al. 2001, ApJ, 559, 1005

Persi, P., Tapia, M., Roth, M., et al. 2003, A\&A, 397, 227

Peters, T., Schleicher, D. R. G., Smith, R. J., Schmidt, W., \& Klessen, R. S. 2014, MNRAS, 442, 3112

Pewsey, A., Neuhäuser, M., \& Ruxton, G. 2013, Circular Statistics in R, EBLSchweitzer (Oxford: Oxford University Press)

Planck Collaboration Int. XXXV. 2016, A\&A, 586, A138

Qin, S.-L., Wu, Y., Huang, M., et al. 2010, ApJ, 711, 399

Qiu, K., Zhang, Q., Menten, K. M., et al. 2014, ApJ, 794, L18

Sanna, A., Reid, M. J., Moscadelli, L., et al. 2009, ApJ, 706, 464

Seifried, D., Banerjee, R., Pudritz, R. E., \& Klessen, R. S. 2012, MNRAS, 423, L40

Stepanovs, D., Fendt, C., \& Sheikhnezami, S. 2014, ApJ, 796, 29

Susa, H., Doi, K., \& Omukai, K. 2015, ApJ, 801, 13

Tan, J. C., Kong, S., Butler, M. J., Caselli, P., \& Fontani, F. 2013, ApJ, 779, 96

Tassis, K., Willacy, K., Yorke, H. W., \& Turner, N. J. 2014, MNRAS, 445, L56

Testi, L., Felli, M., Persi, P., \& Roth, M. 1998, A\&A, 329, 233

Testi, L., Hofner, P., Kurtz, S., \& Rupen, M. 2000, A\&A, 359, L5

Thompson, M. A., Hatchell, J., Walsh, A. J., MacDonald, G. H., \& Millar, T. J. 2006, A\&A, 453, 1003

Troland, T. H., \& Crutcher, R. M. 2008, ApJ, 680, 457

Vaidya, B., Hartquist, T. W., \& Falle, S. A. E. G. 2013, MNRAS, 433, 1258

Vázquez-Semadeni, E., Banerjee, R., Gómez, G. C., et al. 2011, MNRAS, 414, 2511

Vlemmings, W. H. T. 2008, A\&A, 484, 773

Wang, K., Zhang, Q., Testi, L., et al. 2014, MNRAS, 439, 3275

Wardle, J. F. C., \& Kronberg, P. P. 1974, ApJ, 194, 249

Williams, S. J., Fuller, G. A., \& Sridharan, T. K. 2004, A\&A, 417, 115

Zhang, Q., Qiu, K., Girart, J. M., et al. 2014, ApJ, 792, 116

Zhao, B., Caselli, P., Li, Z.-Y., et al. 2016, MNRAS, 460, 2050 